KL-TH $97 / 3$

\title{
Skyrme Sphalerons of an $O(3)-\sigma$ Model and the Calculation of Transition Rates at Finite Temperature
}

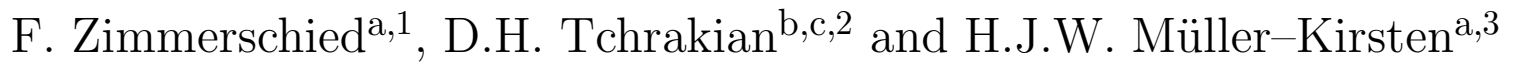 \\ a Department of Physics \\ University of Kaiserslautern, P. O. Box 3049, D 67653 Kaiserslautern, Germany \\ b Department of Mathematical Physics \\ St. Patrick's College, Maynooth, Ireland \\ c School of Theoretical Physics, Dublin Institute for Advanced Studies, \\ 10 Burlington Road, Dublin 4, Ireland
}

\begin{abstract}
The reduced $O(3)-\sigma$ model with an $O(3) \rightarrow O(2)$ symmetry breaking potential is considered with an additional Skyrmionic term, i.e. a totally antisymmetric quartic term in the field derivatives. This Skyrme term does not affect the classical static equations of motion which, however, allow an unstable sphaleron solution. Quantum fluctuations around the static classical solution are considered for the determination of the rate of thermally induced transitions between topologically distinct vacua mediated by the sphaleron. The main technical effect of the Skyrme term is to produce an extra measure factor in one of the fluctuation path integrals which is therefore evaluated using a measure-modified Fourier-Matsubara decomposition (this being one of the few cases permitting this explicit calculation). The resulting transition rate is valid in a temperature region different from that of the original Skyrme-less model, and the crossover from transitions dominated by thermal fluctuations to those dominated by tunneling at the lower limit of this range depends on the strength of the Skyrme coupling.
\end{abstract}

\footnotetext{
${ }^{1} \mathrm{E}$-mail: zimmers@physik.uni-kl.de

${ }^{2} \mathrm{E}$-mail: tchrakian@ailm.may.ie

${ }^{3} \mathrm{E}$-mail: mueller1@gypsy.physik.uni-kl.de
} 


\section{Introduction}

The complicated vacuum structure of quantum field theories involving nonabelian gauge symmetries has been a subject of considerable interest since its consequences have been discussed for the first time [1, 2]. In such theories, besides the usual quantum states built perturbatively on the topologically distinct vacua (characterized by a topological quantity, the winding number), there are nonperturbative phenomena relating these separate perturbative sectors [3]. In particular, there are transitions between the states built on neighbouring vacua which are dominated by different physical processes, depending on the temperature of the system. These processes can be described semiclassically by pseudoparticles, i.e. classical configurations with particle-like properties. The most important example for these effects is the electroweak model in which winding number transitions are related to baryon/lepton number nonconservation [4, 5]. Most calculations, however, are performed in simpler, more manageable "toy models".

This article presents the evaluation of such a transition rate in a special temperature region for a particular but typical model which has the advantage of permitting explicit calculation in a nontrivial case. In this introduction, we therefore first present the general physical background, i.e. the physics of transitions between topologically distinct sectors of a quantum field theory in different temperature regions, and then introduce the particular model which is motivated by its special temperature behaviour, but also by some special technical properties.

\subsection{Transition rates due to tunneling or thermal fluctuations}

Depending on the temperature of the system, transition processes between neighbouring perturbative sectors of a quantum field theory are dominated by different physical processes:

At zero temperature $T=0$, the relevant process is tunneling through the energy barrier separating the neighbouring vacua. In a semiclassical approximation, this tunneling can be described by vacuum instantons [6, 7]. Vacuum instantons are pseudoparticles which are stable classical solutions of the Euclidean Euler-Lagrange equations of the theory with vanishing Euclidean energy as boundary condition, $\mathcal{E}_{\text {inst }}=0$. A semiclassical expansion of the Euclidean path integral around the instanton yields the rate of tunneling through the barrier separating the topologically distinct vacua between which the instanton interpolates as a funtion of imaginary time, used here to describe tunneling. The tree approximation of the instanton tunneling transition rate is thus $\exp \left\{-2 S_{0}\right\}$ where $S_{0}$ is the Euclidean action of the vacuum instanton [8].

At finite temperatures $T>0$, there are thermally excited states in the neighbouring sectors, and starting from some lower temperature limit, transitions due to thermal fluctuations over the separating barrier become relevant. These transitions are described semiclassically by pseudoparticles called sphalerons. These are 
classical solutions of the static Euler-Lagrange equations with finite Euclidean energy $\mathcal{E}_{s p h}$ which are unstable, i.e. the static Gaussian fluctuation operator around these solutions has one negative eigenvalue $\omega_{-}$[9, 10]. Sphalerons can be visualized as "sitting on top" of the energy barrier separating neighbouring vacua. The corresponding semiclassical transition rate is suppressed by the classical Boltzmann factor, $\exp \left\{-\mathcal{E}_{\text {sph }} \beta_{T}\right\}$ which one obtains as the tree approximation of the partition function path integral which again involves the use of complex time, now in the convenient formalism of quantum field theory at finite temperature [11, 13]. Comparing the leading exponential factors of the semiclassical expansion around instantons and sphalerons, respectively, one finds that thermal fluctuations dominate vacuum tunneling in the transition rate for temperatures $T>T_{0}=: \frac{1}{k_{B}} \frac{\mathcal{E}_{s p h}}{2 S_{0}}$.

A third transition process which has been discussed intensively in recent literature [14, 15] is tunneling from (thermally) excited states, described by a Boltzmann average over nonvacuum instantons which are classical solutions of the Euclidean EulerLagrange equations with Euclidean energy $\mathcal{E}_{\text {inst }}>0$ as boundary condition [16, 17]. These solutions are periodic in the imaginary time coordinate with energy dependent period $\hat{\tau}=\hat{\tau}\left(\mathcal{E}_{\text {inst }}\right)$ (which is why they are usually called "periodic instantons"). In order to apply the Boltzmann average over the periodic instantons, one has to satisfy the saddle point condition $\hat{\tau}\left(\mathcal{E}_{\text {inst }}\right) \equiv \beta_{T}$, so that only periodic instantons with period equal to the inverse temperature dominate the nonvacuum tunneling transition rate. The Euclidean action of the periodic instanton can thus be written $S_{\text {inst }}\left(\beta_{T}\right)$, and the usual relation $\mathcal{E}_{\text {inst }}=\frac{S_{\text {inst }}\left(\beta_{T}\right)}{\beta_{T}}$ holds. The two different uses of complex time (in tunneling and in thermodynamics) thus merge together in the case of the periodic instanton.

For $\mathcal{E}_{\text {inst }} \rightarrow \mathcal{E}_{\text {sph }}$ the imaginary time dependence vanishes and the periodic instanton reduces to the sphaleron (being time periodic with any period, i.e. static) with Euclidean action $S_{s p h}\left(\beta_{T}\right)=\mathcal{E}_{\text {sph }} \beta_{T}$. The second limit $\mathcal{E}_{\text {inst }} \rightarrow 0$ which should reduce the periodic instanton to the vacuum one shows that there are two different types of periodic instantons [15]: Those with $\hat{\tau}\left(\mathcal{E}_{\text {inst }}\right) \stackrel{\mathcal{E}_{\text {inst }}}{\longrightarrow} 0$ where one has localised vacuum instantons with infinite period and those with $\hat{\tau}\left(\mathcal{E}_{\text {inst }}\right) \stackrel{\mathcal{E}_{\text {inst }} \rightarrow 0}{\longrightarrow} 0$ where localised vacuum instantons do not exist. Nonetheless the well-known method of constrained instantons [16, 18] (which may be interpreted as the zero period limit of this second type of periodic instantons) allows vacuum tunneling even in this case. In either case the dilute gas approximation works and one has $S_{\text {inst }}\left(\beta_{T}\right) \stackrel{\beta_{T} \rightarrow 0}{\longrightarrow} 2 S_{0}$.

It is now an interesting and important question to ask how the temperature domains in which the different physical processes (vacuum tunneling, thermal tunneling, thermal fluctuations) dominate the transition rate, can be continued into each other. The crucial point here is the crossover from predominantly thermal fluctuations to predominantly (vacuum or thermally excited) tunneling. Near the sphaleron energy, $\mathcal{E}_{\text {sph }}-\mathcal{E}_{\text {inst }} \ll \mathcal{E}_{\text {inst }}$, the periodic instanton can be written as a sum of the sphaleron and oscillations in its eigenmode with negative eigenvalue $\omega_{-}$. In the limit $\mathcal{E}_{\text {inst }} \rightarrow \mathcal{E}_{\text {sph }}$, the period of oscillation approaches $\hat{\tau}_{s p h}:=\frac{2 \pi}{\omega_{-}}=: \frac{1}{k_{B} T^{(-)}}$, the 


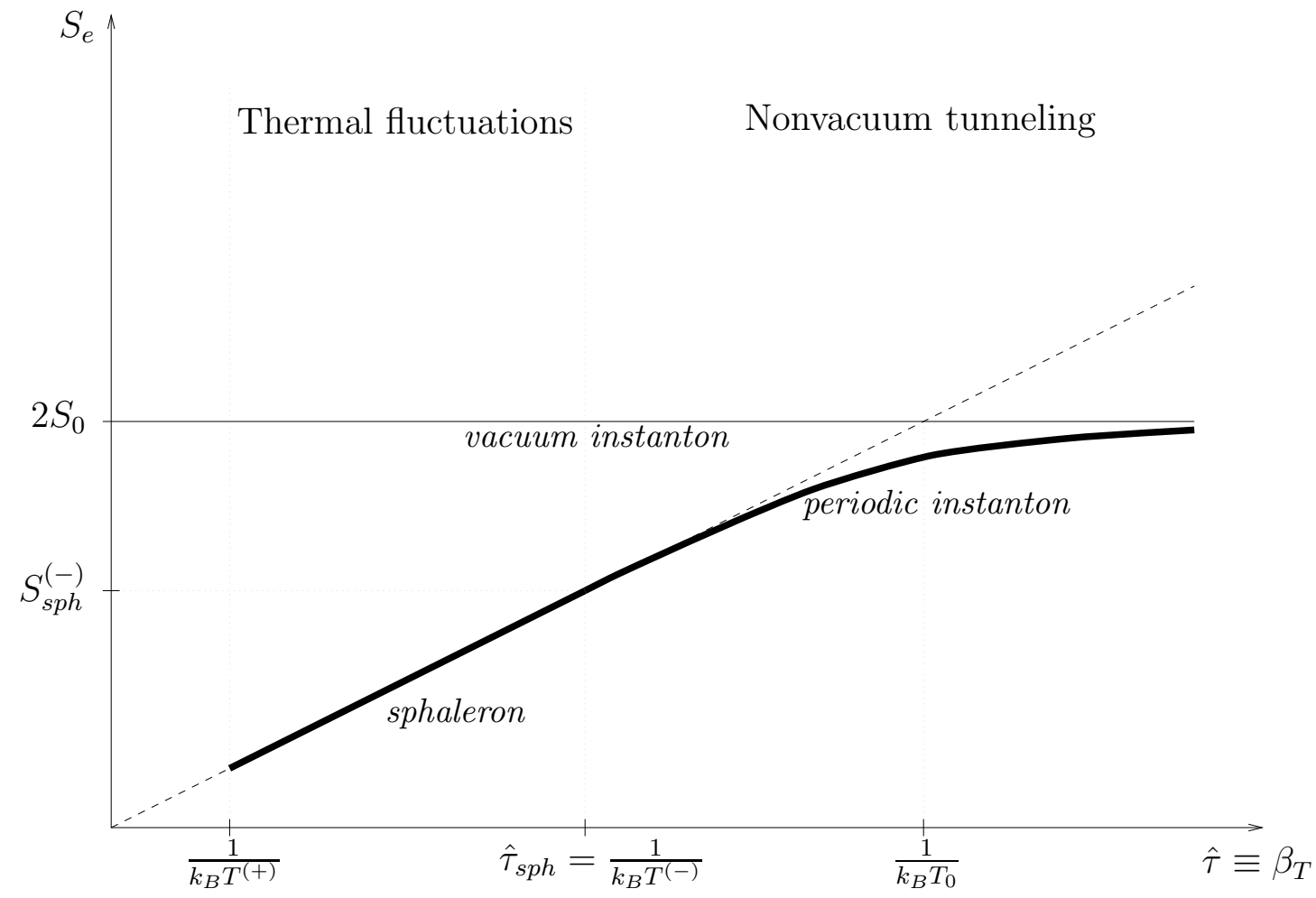

Figure 1: Type I theory (the solution that dominates the transition rate is indicated by the thick line)

periodic instanton thereby merging into the sphaleron which has Euclidean action $S_{s p h}\left(\hat{\tau}_{s p h}\right)=S_{s p h}^{(-)}$at this lower temperature bound. Depending on the model, the period $\hat{\tau}\left(\mathcal{E}_{\text {inst }}\right)$ for $\mathcal{E}_{\text {inst }}<\mathcal{E}_{\text {sph }}$ may be smaller or larger than $\hat{\tau}_{s p h}$.

This fact, together with the two types of low energy behaviour of the periodic instantons, results in two different simple types of theories with continuous crossover between the temperature domains with different dominant processes [15]. In type I theories, we have localised instantons, $\hat{\tau}\left(\mathcal{E}_{\text {inst }}\right) \stackrel{\mathcal{E}_{\text {inst }} \rightarrow 0}{\longrightarrow} \infty$, the period $\hat{\tau}$ is always greater than $\hat{\tau}_{s p h}$, thus $\frac{\partial^{2} S_{\text {inst }}\left(\beta_{T}\right)}{\partial \beta_{T}^{2}}=\frac{\partial \mathcal{E}_{\text {inst }}}{\partial \beta_{T}}<0$, and $2 S_{0}>S_{s p h}^{(-)}$. Then $T_{0}<T^{(-)}$, thermal fluctuations dominate for $T>T^{(-)}$and nonvacuum tunneling from thermally excited states dominates for $T<T^{(-)}$, and is suppressed by $\exp \left\{-S_{\text {inst }}\left(\beta_{T}\right)\right\}$. The crossover between the two regions is smooth. This situation is shown in Fig. 11. Type II theories have nonlocalised vacuum instantons, $\hat{\tau}\left(\mathcal{E}_{\text {inst }}\right) \stackrel{\mathcal{E}_{\text {inst }} \rightarrow 0}{\longrightarrow} 0$, the period $\hat{\tau}$ is always smaller than $\hat{\tau}_{s p h}$, thus $\frac{\partial^{2} S_{\text {inst }}\left(\beta_{T}\right)}{\partial \beta_{T}^{2}}=\frac{\partial \mathcal{E}_{\text {inst }}}{\partial \beta_{T}}>0$, and $2 S_{0}<S_{s p h}^{(-)}$. This yields $T^{(-)}<T_{0}$ : Thermal fluctuations dominate for $T>T_{0}$, vacuum tunneling for $T<T_{0}$ and the crossover is sharp as shown in Fig. 2 .

Well known examples are the $1+1$ dimensional Higgs model [16, 19] for a type I theory and the reduced nonlinear $O(3)-\sigma$ model [20, 14] for a type II theory. Both are 


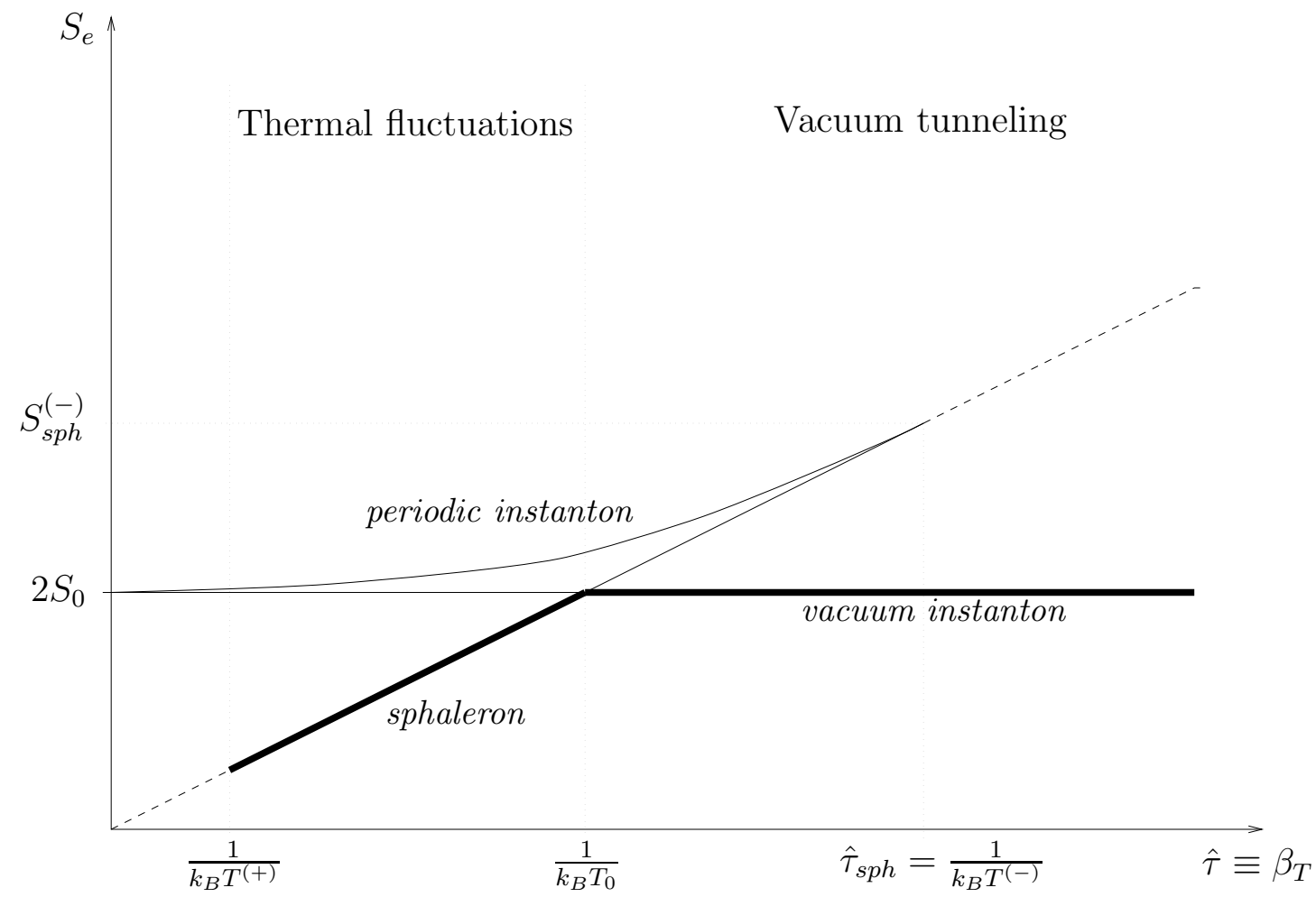

Figure 2: Type II theory (the solution that dominates the transition rate is indicated by the thick line)

prominent toy models for the winding number transition in the electroweak model. But the electroweak theory seems to be neither of type I nor of type II: It has no localised instantons which disagrees with type I properties, and one estimates $2 S_{0}>\mathcal{E}_{\text {sph }} \hat{\tau}_{\text {sph }}$ for the electroweak constrained instantons, so that the theory is not of type II.

To understand this special property of the electroweak model which is not shared by the usual toy models, it is interesting to study simple low dimensional models which also can not be classified under the two types above, although such models may not be good toy models for the electroweak theory from other points of view. One of these models of recent interest [15] is the reduced nonlinear $O(3)-\sigma$ model in $1+1$ dimensions with an added Skyrme term [21].

\subsection{The reduced $O(3)-\sigma$ model with Skyrme term and its motivation}

The model we deal with here is defined by the Lagrangian density

$$
\mathcal{L}\left(\phi^{a}, \partial_{\mu} \phi^{a}\right)=\frac{\lambda_{2}}{8}\left(\partial_{[\mu} \phi^{a} \partial_{\nu]} \phi^{b}\right)\left(\partial^{[\nu} \phi^{a} \partial^{\mu]} \phi^{b}\right)+\frac{\lambda_{1}}{2}\left(\partial_{\mu} \phi^{a} \partial^{\mu} \phi^{a}\right)-\lambda_{0} V\left(\phi^{3}\right)
$$




$$
\begin{aligned}
& =\frac{\lambda_{2}}{2}\left[\dot{\vec{\phi}} \wedge \vec{\phi}^{\prime}\right]^{2}+\frac{\lambda_{1}}{2}\left[(\dot{\vec{\phi}})^{2}-\left(\vec{\phi}^{\prime}\right)^{2}\right]-\lambda_{0} V\left(\phi^{3}\right) \\
& =\lambda_{1}\left\{\frac{\nu^{2}}{2}\left[(\dot{\vec{\phi}})^{2}\left(\vec{\phi}^{\prime}\right)^{2}-\left(\dot{\vec{\phi}} \cdot \vec{\phi}^{\prime}\right)^{2}\right]+\frac{1}{2}\left[(\dot{\vec{\phi}})^{2}-\left(\vec{\phi}^{\prime}\right)^{2}\right]-\mu^{2} V\left(\phi^{3}\right)\right\}
\end{aligned}
$$

with

$$
\nu^{2}:=\frac{\lambda_{2}}{\lambda_{1}}, \quad \mu^{2}:=\frac{\lambda_{0}}{\lambda_{1}}
$$

in Minkowski spacetime $\left(x^{0}, x^{1}\right)=(t, x)$ with fields $\vec{\phi}=\left(\phi^{a}\right), a=1,2,3$ constrained by $\phi^{a} \phi^{a}=1$ (we do not add a Lagrange multiplier since we will always work with parametrisations respecting the constraint). This Lagrangian differs from that of the usual nonlinear $\sigma$ model, $\mathcal{L}=\frac{1}{2 g^{2}}\left(\partial_{\mu} \phi^{a} \partial^{\mu} \phi^{a}\right)$ [22] (comparing the kinetic terms of the two Lagrangians, the two couplings $\frac{1}{g^{2}}=\lambda_{1}$ have to be identified) with $\phi^{a} \phi^{a}=1$ by two additional terms. The explicit $O(3) \rightarrow O(2)$ symmetry breaking potential (which we choose to be $V\left(\phi^{3}\right)=1-\phi^{3}$ ), being necessary to produce a sphaleron in the theory (there is no mechanism of spontaneous symmetry breaking for $\sigma$ models), and the Skyrme term with coupling $\nu^{2}$. Setting $\nu^{2}=0$ reduces this model to the well-known reduced nonlinear $O(3)-\sigma$ model which provides good model predictions for the electroweak theory [20]. The latter is the original Skyrme-less model which has no localised instantons and is a type II theory [14].

Adding a Skyrme term changes this last aspect completely. First, there is a localised vacuum instanton $\varphi_{\text {inst }}$ in the Skyrmed theory [23] which can be verified numerically [21]. Its action is [15]

$$
S_{0}=4 \pi \lambda_{1}+\mathcal{O}\left(\lambda_{1} \nu \mu\right) .
$$

In this model the sphaleron solution $\varphi_{s p h}$ is not affected by the Skyrme term because it solves the static Euler-Lagrange equations and the Skyrme term is purely dynamical. But the fluctuation spectrum around the sphaleron is modified and therefore also the one-loop quantum correction in the semiclassical transition rate. Moreover, the temperature range of validity of this calculation changes. In particular, the temperatures $T_{0}$ and $T^{(-)}$will depend on $\nu$.

It is the purpose of this investigation to calculate the thermal transition rate in a semiclassical one-loop expansion around the sphaleron in this Skyrmed theory. This involves the evaluation of fluctuation determinants, and one main motivation of this paper (besides knowing that the Skyrmed reduced nonlinear $O(3)-\sigma$ model is a theory neither of type I nor of type II) is that these evaluations can be performed explicitly, and one can finally see clearly the role of the Skyrme term in the thermal transition rate.

Moreover, the technical aspects of the calculational difficulties which the Skyrme term gives rise to are presented in detail. These techniques should be of interest also in the context of more physically motivated models like the three dimensional $\sigma$ model which is motivated by QCD. In the case of the latter, the hope is that the 
loop contributions could give rise to Skyrme-like terms in the action which would stabilize the Skyrmion [24].

The layout of the paper is as follows: In Section 8 the notion of a sphaleron is reviewed. The NCL technique is discussed in the context of the Skyrmed $O(3)-\sigma$ model, the sphaleron solution is derived and its instability is discussed in terms of the static Gaussian fluctuation operators.

The embedding of the sphaleron of the reduced $O(3)-\sigma$ model into the full Euclidean theory is described in Section 3 where the thermodynamical formalism yielding the transition rate is reviewed, using the path integral version of quantum statistics [25]. What is quantized are the fluctuations parallel and perpendicular to the vacuum and the sphaleron, respectively. Since these fluctuations are dynamical quantities, they may involve the (kinetic) Skyrme term. The effect of the latter on the model is to produce an extra measure factor which modifies the scalar product in the Hilbert space of the perpendicular fluctuations around the sphaleron. Therefore the evaluation of the path integrals is also considered in some detail in this Section, taking care of the path integral measures.

Sections 4 and 5 deal with the fluctuations around the vacuum and the parallel fluctuations around the sphaleron which are already well known since there is no influence of the Skyrme term on this part of the theory [20].

The new effects of the Skyrme measure factor on the perpendicular fluctuations around the sphaleron are investigated in Section 6 .

In Section 0 the results of the calculations are summarized to yield the thermally activated transition rate. The Skyrme-less limit is discussed, as well as the temperature range of validity of the calculation.

The physical implications of this analysis are then discussed in the concluding Section 8.

Finally, some mathematical steps and calculational details are given in the $A p$ pendices.

\section{Saddle-point configurations of the static energy functional}

The solutions we consider are static, $\vec{\phi}=\vec{\phi}(x)$, and result as extrema of the static energy functional

$$
\mathcal{E}[\vec{\phi}]=\int \mathcal{H}_{\text {stat }} d x
$$

where $\mathcal{H}_{\text {stat }}$ is the static part of the Hamiltonian, defined as the 00 -component $\mathcal{H}=$

$T_{00}$ of the energy-momentum tensor $T_{\mu \nu}:=\Pi_{\mu}^{a} \phi_{\nu}^{a}-g_{\mu \nu} \mathcal{L}, \Pi_{\mu}^{a}:=\frac{\partial \mathcal{L}}{\partial\left(\partial^{\mu} \phi^{a}\right)}$, or obtained alternatively from the well-known formula

$$
\mathcal{H}:=\vec{\Pi} \dot{\vec{\phi}}-\mathcal{L}, \quad \vec{\Pi}:=\frac{\partial \mathcal{L}}{\partial \dot{\vec{\phi}}}
$$


Although one cannot write down the Hamiltonian in terms of fields and conjugate momenta explicitly, it is easy to see that

$$
\mathcal{H}_{\text {stat }}=\frac{\lambda_{1}}{2}\left(\vec{\phi}^{\prime}\right)^{2}+\lambda_{0} V\left(\phi^{3}\right)
$$

For the special potential $V\left(\phi^{3}\right)=1-\phi^{3}$, the vacuum configuration of $\mathcal{E}[\vec{\phi}]$ is thus given by $\vec{\varphi}_{0}=(0,0,1)$.

We parametrize the fields so that they satisfy the constraint $\vec{\phi}^{2}=1$. There are at least three appropriate parametrizations of the static fields,

$$
\begin{aligned}
\vec{\phi}(x) & =\frac{1}{\sqrt{1+\left(f^{2}(x)\right)^{2}}}\left(\begin{array}{c}
\sin f^{1}(x) \\
f^{2}(x) \\
\cos f^{1}(x)
\end{array}\right) \\
\vec{\phi}(x) & =\left(\begin{array}{c}
\sin g^{1}(x) \cos g^{2}(x) \\
\sin g^{1}(x) \sin g^{2}(x) \\
\cos g^{1}(x)
\end{array}\right) \\
\vec{\phi}(x) & =\left(\begin{array}{c}
\sin h^{1}(x) \sin h^{2}(x) \\
\sin h^{1}(x) \cos h^{1}(x)\left(\cos h^{2}(x)-1\right) \\
\sin ^{2} h^{1}(x) \cos h^{2}(x)+\cos ^{2} h^{1}(x)
\end{array}\right)
\end{aligned}
$$

In terms of the new parameter fields $\vec{f}(x), \vec{g}(x)$ and $\vec{h}(x)$, the static energy functional (四) is $\mathcal{E}[\phi]=\mathcal{E}_{f}[\vec{f}]=\mathcal{E}_{g}[\vec{g}]=\mathcal{E}_{h}[\vec{h}]$ with

$$
\begin{aligned}
\mathcal{E}_{f}[\vec{f}] & =\int\left[\lambda_{1}\left\langle\vec{f}^{\prime}(x), F(\vec{f}(x)) \vec{f}^{\prime}(x)\right\rangle+\lambda_{0} V\left(\frac{\cos f^{1}(x)}{\sqrt{1+\left(f^{2}(x)\right)^{2}}}\right)\right] d x \\
\mathcal{E}_{g}[\vec{g}] & =\int\left[\lambda_{1}\left\langle\vec{g}^{\prime}(x), G(\vec{g}(x)) \vec{g}^{\prime}(x)\right\rangle+\lambda_{0} V\left(\cos g^{1}(x)\right)\right] d x \\
\mathcal{E}_{h}[\vec{h}] & =\int\left[\lambda_{1}\left\langle\vec{h}^{\prime}(x), H(\vec{h}(x)) \vec{h}^{\prime}(x)\right\rangle+\lambda_{0} V\left(\sin ^{2} h^{1}(x) \cos h^{2}(x)+\cos ^{2} h^{1}(x)\right)\right] d x
\end{aligned}
$$

with the matrices

$$
\begin{aligned}
F(\vec{f}) & =\left(\begin{array}{cc}
\frac{1}{1+\left(f^{2}\right)^{2}} & 0 \\
0 & \frac{1}{\left(1+\left(f^{2}\right)^{2}\right)^{2}}
\end{array}\right) \\
G(\vec{g}) & =\left(\begin{array}{cc}
1 & 0 \\
0 & \sin ^{2} g^{1}
\end{array}\right) \\
H(\vec{h}) & =\left(\begin{array}{cc}
\cos ^{2} h^{1} \sin ^{2} h^{2}+\left(1-\cos h^{2}\right)^{2} & \sin h^{1} \cos h^{1} \sin h^{2} \\
\sin h^{1} \cos h^{1} \sin h^{2} & \sin ^{2} h^{1}
\end{array}\right)
\end{aligned}
$$

The $\vec{h}$-parametrization is usually used to find the saddle-point of the static energy functional (4) with the special potential $V\left(\phi^{3}\right)=1-\phi^{3}$. Treating $h^{1}(x)$ as a constant 
parameter $h^{1}(x) \equiv \eta$ and writing $h^{2}(x)=f(x)$, the parametrization (9) yields a noncontractible loop (NCL) 26]

$$
\vec{\phi}_{\eta}(x)=\left(\begin{array}{c}
\sin \eta \sin f(x) \\
\sin \eta \cos \eta(\cos f(x)-1) \\
\sin ^{2} \eta \cos f(x)+\cos ^{2} \eta
\end{array}\right)
$$

in the space of static field configurations on which $\mathcal{E}$ is defined. The NCL starts and ends in the vacuum. The energy of the configurations along this loop is given by

$$
\mathcal{E}\left[\vec{\phi}_{\eta}\right]=\lambda_{1} \sin ^{2} \eta \int\left[\frac{1}{2}\left(f^{\prime}(x)\right)^{2}+\mu^{2}(1-\cos f(x))\right] d x
$$

which has a maximum in the $\eta$-direction for $\eta=\frac{\pi}{2}$. Minimizing the resulting energy function by variation of $f(x)$,

$$
\delta \mathcal{E}\left[\vec{\phi}_{\left(\eta=\frac{\pi}{2}\right)}\right]=0 \quad \Longrightarrow \quad \lambda_{1} \frac{\delta}{\delta f(y)} \int\left[\frac{1}{2}\left(f^{\prime}(x)\right)^{2}+\mu^{2}(1-\cos f(x))\right] d x=0
$$

yields the well-known Sine-Gordon equation

$$
f^{\prime \prime}(x)-\mu^{2} \sin f(x)=0, \quad \mu^{2}:=\frac{\lambda_{0}}{\lambda_{1}} .
$$

Assuming as boundary condition that for $|x| \rightarrow \infty, \vec{\phi}(x)$ approaches the vacuum $\vec{\varphi}_{0}$, i. e. $f(x) \stackrel{|x| \rightarrow \infty}{\longrightarrow} 0,2 \pi$, the solution of (19) is

$$
f(x)=4 \arctan \left(e^{ \pm \mu\left(x-x_{0}\right)}\right) .
$$

From the NCL technique [26], we expect the classical solution $\vec{\varphi}$ which in this parametrization is given by $\eta=\frac{\pi}{2}, f(x)$, i.e. $\vec{\varphi}_{s p h}(x)=\vec{\varphi}_{1}(x)=(\sin f(x), 0, \cos f(x))$, to be a saddle-point of the energy functional $\mathcal{E}[\vec{\phi}]$. This can be verified by considering the second variational derivative of $\mathcal{E}[\vec{\phi}]$ which should be taken in a convenient parametrization of $\vec{\phi}$ to avoid separate treatment of the $\vec{\phi}^{2}=1$ constraint. Using the parametrizations (77,8, 91) we thus have to analyse the eigenvalues of the second variation of $\mathcal{E}_{f}[\vec{g}], \mathcal{E}_{g}[\vec{f}]$ or $\mathcal{E}_{h}[\vec{h}]$ taken at the classical solution $\vec{\varphi}_{1}(x)$, which means $f^{1}(x)=f(x), f^{2}(x)=0 ; g^{1}(x)=f(x), g^{2}(x)=0$ or $h^{1}(x)=\frac{\pi}{2}, h^{2}(x)=f(x)$ respectively:

$$
\begin{gathered}
\int\left[\frac{\delta^{2} \mathcal{E}_{f}[\vec{f}]}{\delta f^{i}(x) \delta f^{j}\left(x^{\prime}\right)}\right]_{\vec{\varphi}} \psi_{f}^{j}\left(x^{\prime}\right) d x^{\prime}=\omega^{2} \psi_{f}^{i}(x) \\
\int\left[\frac{\delta^{2} \mathcal{E}_{g}[\vec{g}]}{\delta g^{i}(x) \delta g^{j}\left(x^{\prime}\right)}\right]_{\vec{\varphi}} \psi_{g}^{j}(x) d x^{\prime}=\omega^{2} \psi_{g}^{i}(x) \\
\int\left[\frac{\delta^{2} \mathcal{E}_{h}[\vec{h}]}{\delta h^{i}(x) \delta h^{j}\left(x^{\prime}\right)}\right]_{\vec{\varphi}} \psi_{h}^{j}(x) d x^{\prime}=\omega^{2} \psi_{h}^{i}(x)
\end{gathered}
$$


with $i, j=1,2$. These eigenvalue equations are equivalent. The $\vec{f}$-parametrization yields directly decoupled equations in a simple form. Eqs. (23) have to be decoupled, whereas (22) are decoupled, but can be simplified by a substitution. This is why the $\vec{f}$-parametrization is preferred in the following, where we will modify our notations slightly to $\vec{f}=\left(f^{(\|)}, f^{(\perp)}\right)$ to denote the two parameter fields. We will see in the next section that $f^{(\|)}$describes fluctuations parallel and $f^{(\perp)}$ those perpendicular to the sphaleron $\vec{\varphi}_{1}(x)$. In this new notation, each of the eigenvalue equations (21 23) may be written as a system of two decoupled static fluctuation equations, i.e.

$$
\begin{aligned}
\hat{\mathcal{H}}_{\|} \psi^{(\|)}(x)=\frac{\left(\omega^{(\|)}\right)^{2}}{\lambda_{1}} \psi^{(\|)}(x), & \hat{\mathcal{H}}_{\|}=-\frac{\partial^{2}}{\partial x^{2}}+\mu^{2}\left\{1-2 \operatorname{sech}^{2}(\mu x)\right\} \\
\hat{\mathcal{H}}_{\perp} \psi^{(\perp)}(x)=\frac{\left(\omega^{(\perp)}\right)^{2}}{\lambda_{1}} \psi^{(\perp)}(x), & \hat{\mathcal{H}}_{\perp}=-\frac{\partial^{2}}{\partial x^{2}}+\mu^{2}\left\{1-6 \operatorname{sech}^{2}(\mu x)\right\} .
\end{aligned}
$$

The second equation (25) has one negative eigenvalue $\left(\omega^{(\perp)}\right)^{2}=-3 \mu^{2}$; thus the static solution $\vec{\varphi}_{1}(x)$ is always unstable in the space of static field configurations. It is important to note that we never left this space. The sphaleron is a static object, and one can not decide whether it is Minkowskian or Euclidean.

The notion of a "static fluctuation operator" reflects the fact that one can derive the stability operator (10) (which is usually called the "static Gaussian fluctuation operator") by expanding $\mathcal{E}_{f}[\vec{f}]$ in small "fluctuations" (which are static in this context!) around the classical solutions, and similary for the other parametrizations. These fluctuations become dynamical quantities if one embeds the sphaleron into the full, time-dependent theory, either in its Minkowskian version (II) or in the Euclidean version which one obtains from the former by a Wick rotation in the time coordinate, $t \mapsto \tau=-i t$. As the sphaleron itself does not depend on time even after the embedding into the full, e.g. Euclidean theory, one may impose periodic boundary conditions in the imaginary time coordinate on the fields. Then one can treat the result as a field theory at finite temperature which is defined via the imaginary time period $\beta_{T}=\frac{1}{k_{B} T}$ (we set $k_{B}=1$ ), and the usual path integral formalism of quantum statistics [25] is applicable, including the calculation of thermally activated transitions over energy barriers.

\section{Finite Temperature: Sphalerons in Euclidean spacetime}

From the general theory of thermal transition rates [18, 27], we know that transitions due to fluctuations at finite temperature may be decribed by the imaginary part of the free energy $F$,

$$
\Gamma=\frac{|\varrho|}{\pi} \operatorname{Im}\left(\beta_{T} F\right)
$$


where $\varrho$ is a damping constant. As usual, $F$ is related to the partition function $\mathcal{Z}$ by $\mathcal{Z}=e^{-\beta_{T} F}$, so that the partition function of the system at temperature $T$ has to be computed, using the path integral version of quantum statistics [25] [11], i.e.

$$
\mathcal{Z}=\int \prod_{i=1}^{3}\left[\mathrm{~d} \phi^{i}(\tau, x)\right] \prod_{i=1}^{3}\left[\mathrm{~d} \Pi^{i}(\tau, x)\right] \delta(|\vec{\phi}|-1) e^{-S_{e}[\vec{\phi}, \vec{\Pi}]} .
$$

Here we start from the Hamiltonian version of the partition function path integral 111] (we emphasize this also by the use of the symbol [d...] for the path measures): To obtain the Lagrangian version, one has to integrate out the momenta in the Euclidean action of the system,

$$
S_{e}[\vec{\phi}, \vec{\Pi}]=\int_{0}^{\beta_{T}} d \tau \int_{-\infty}^{\infty} d x\left(-i \vec{\Pi} \dot{\vec{\phi}}+\mathcal{H}\left(\vec{\phi}, \vec{\phi}^{\prime}, \vec{\Pi}\right)\right)
$$

where $\mathcal{H}$ is the Hamiltonian density (5) of the system.

If the kinetic term of a field theory Lagrangian is quadratic in the time derivatives, e. g. for a field $w(\tau, x)$

$$
\mathcal{L}\left(w, w^{\prime}, \dot{w}\right)=\frac{1}{2} M(\dot{w})^{2}-\mathcal{U}\left(w, w^{\prime}\right)
$$

(which means $\vec{\pi}=M \dot{w}$ ), the momentum integration (which in this case is a standard Gaussian integral) yields the well-known Feynman formula 25

$$
\mathcal{Z}=\int \mathcal{D}\{w\} e^{-\int_{0}^{\beta_{T}} d \tau \int_{-\infty}^{\infty} d x \mathcal{L}_{e}\left(w, w^{\prime}, \dot{w}\right)}
$$

$(\mathcal{D}\{\ldots\}$ denoting Lagrangian path measures which depend on the mass $M)$ with the Euclidean Lagrange density

$$
\mathcal{L}_{e}\left(w, w^{\prime}, \dot{w}\right)=\frac{1}{2} M(\dot{w})^{2}+\mathcal{U}\left(w, w^{\prime}\right)
$$

where dots now denote derivatives with respect to $\tau$. Of course one can also compute the Euclidean Lagrangian of our Skyrme model from its Minkowskian version (回),

$$
\mathcal{L}_{e}\left(\vec{\phi}, \vec{\phi}^{\prime}, \dot{\vec{\phi}}\right)=\lambda_{1}\left\{\nu^{2}\left[(\dot{\vec{\phi}})^{2}\left(\vec{\phi}^{\prime}\right)^{2}-\left(\dot{\vec{\phi}} \cdot \vec{\phi}^{\prime}\right)^{2}\right]+\frac{1}{2}\left[(\dot{\vec{\phi}})^{2}+\left(\vec{\phi}^{\prime}\right)^{2}\right]+\mu^{2} V\left(\phi^{3}\right)\right\}
$$

but we can not simply use it in formula (28) since the $\dot{\vec{\phi}}$-dependence in (11) is quadratic but involves a field-dependent mass factor, so that the momentum integration has to be analysed carefully as it will yield a measure factor [12.

Here we perform a perturbative evaluation of $\mathcal{Z}$ around two extrema (there may certainly be more contributions): $\mathcal{Z} \approx \mathcal{Z}_{0}+\mathcal{Z}_{1}$. $\mathcal{Z}_{0}$ is evaluated around the vacuum $\vec{\varphi}_{0}=(0,0,1)$ and thus contains the usual perturbative vacuum fluctuations. We know that this part has to be real. $\mathcal{Z}_{1}$ is evaluated around the sphaleron $\vec{\varphi}(x)$ which 
is also an extremum of the Euclidean action $S_{e}[\vec{\phi}]$. We expect this part to yield an imaginary contribution to the partition function since we want to describe thermal transitions by the sphaleron. Assuming $\left|\mathcal{Z}_{1}\right| \ll \mathcal{Z}_{0}$, we may write

$$
\operatorname{Im}\left(\beta_{T} F\right)=-\operatorname{Im}(\ln \mathcal{Z}) \approx-\operatorname{Im}\left[\ln \left(\mathcal{Z}_{0}\left(1+\frac{\mathcal{Z}_{1}}{\mathcal{Z}_{0}}\right)\right)\right] \approx-\frac{1}{\mathcal{Z}_{0}} \operatorname{Im}\left(\mathcal{Z}_{1}\right) .
$$

The evaluation around each of the two classical solutions $\vec{\varphi}_{i}, i=0,1$ is done in a parametrization similar to (8), but now with spacetime dependent parameter fields $\vec{f}(\tau, x)=\left(f^{(\|)}(\tau, x), f^{(\perp)}(\tau, x)\right):$

$$
\vec{\phi}(\tau, x)=\frac{1}{\sqrt{1+\left(f^{(\perp)}(\tau, x)\right)^{2}}}\left(\begin{array}{c}
\sin f^{(\|)}(\tau, x) \\
f^{(\perp)}(\tau, x) \\
\cos f^{(\|)}(\tau, x)
\end{array}\right)
$$

In terms of the parameter fields $\vec{f}(\tau, x)$, the classical solutions $\vec{\varphi}_{i}(x)$ are given by $f^{(\|)}(\tau, x)=f_{i}(x), f^{(\perp)}(\tau, x)=0$ with $f_{0}(x)=0, f_{1}(x)=f(x)=4 \arctan \left(e^{ \pm \frac{x-x_{0}}{\sqrt{\mu}}}\right)$. We add fluctuations $\vec{v}(\tau, x)=\left(v^{(\|)}(\tau, x), v^{(\perp)}(\tau, x)\right)$ to both parameter fields to obtain the fluctuation ansatz

$$
\begin{aligned}
\vec{\phi}(\tau, x) & \frac{1}{\sqrt{1+\left(v^{(\perp)}(\tau, x)\right)^{2}}}\left(\begin{array}{c}
\sin \left(f_{i}(x)+v^{(\|)}(\tau, x)\right) \\
v^{(\perp)}(\tau, x) \\
\cos \left(f_{i}(x)+v^{(\|)}(\tau, x)\right)
\end{array}\right) \\
\approx & \left(\begin{array}{c}
\sin f_{i}(x)+v^{(\|)}(\tau, x) \cos f_{i}(x)-\frac{1}{2}\left(\left(v^{(\perp)}(\tau, x)\right)^{2}+\left(v^{(\|)}(\tau, x)\right)^{2}\right) \sin f_{i}(x) \\
v^{(\perp)}(\tau, x) \\
\cos f_{i}(x)-v^{(\|)}(\tau, x) \sin f_{i}(x)-\frac{1}{2}\left(\left(v^{(\perp)}(\tau, x)\right)^{2}+\left(v^{(\|)}(\tau, x)\right)^{2}\right) \cos f_{i}(x)
\end{array}\right) \\
& +\mathcal{O}\left(\left(v^{(\|)}, v^{(\perp)}\right)^{3}\right) \\
= & \left.\vec{\varphi}_{i}(x)+(\vec{\psi}(\vec{v}))\right)(\tau, x)+\mathcal{O}\left(\left(v^{(\|)}, v^{(\perp)}\right)^{3}\right)
\end{aligned}
$$

(One should note that we are considering time dependent fluctuations!). We do not attach indices to the fluctuations since it is clear that the fluctuations are different for $\vec{\varphi}_{0}$ and $\vec{\varphi}_{1}$.

Here,

$$
\begin{aligned}
(\vec{\psi}(\vec{v})) & (\tau, x) \\
= & \left(\begin{array}{r}
v^{(\|)}(\tau, x) \cos f_{i}(x)-\frac{1}{2}\left(\left(v^{(\perp)}(\tau, x)\right)^{2}+\left(v^{(\|)}(\tau, x)\right)^{2}\right) \sin f_{i}(x) \\
v^{(\perp)}(\tau, x) \\
-v^{(\|)}(\tau, x) \sin f_{i}(x)-\frac{1}{2}\left(\left(v^{(\perp)}(\tau, x)\right)^{2}+\left(v^{(\|)}(\tau, x)\right)^{2}\right) \cos f_{i}(x)
\end{array}\right)
\end{aligned}
$$


are the fluctuations around the solution ansatz $\vec{\varphi}_{i}(x)=\left(\sin f_{i}(x), 0, \cos f_{i}(x)\right)$, constructed such that they respect the sigma model constraint $\vec{\phi}^{2}=1$ (at least up two second order in $\vec{\psi}(\vec{v}))$. They are parametrized in terms of two fluctuation functions $\vec{v}(\tau, x)=\left(v^{(\perp)}(\tau, x), v^{(\|)}(\tau, x)\right)$ which represent the two dimensions of a unit sphere.

We now insert (36) into the Lagrangian (11) and expand it to the mentioned second order in the fluctuations which yields the linearized Lagrangian (around the classical solution $\vec{\varphi}_{i}$ )

$$
\tilde{\mathcal{L}}_{i}\left(\vec{v}, \vec{v}^{\prime}, \dot{\vec{v}}\right)=\lambda_{1}\left\{\frac{1}{2}\left[\left\{1+\nu^{2}\left(f_{i}^{\prime}(x)\right)^{2}\right\}\left(\dot{v}^{(\perp)}\right)^{2}+\left(\dot{v}^{(\|)}\right)^{2}\right]-\mathcal{U}_{i}\left(\vec{v}, \vec{v}^{\prime}\right)\right\}
$$

where

$$
\begin{aligned}
\mathcal{U}_{i}\left(\vec{v}, \vec{v}^{\prime}\right)= & \frac{1}{2}\left(\vec{v}^{\prime}\right)^{2}+\left[\frac{1}{2}\left(f_{i}^{\prime}(x)\right)^{2}+\mu^{2} V\left(\cos f_{i}(x)\right)\right] \\
& +\left[f_{i}^{\prime}(x)\left(v^{(\|)}\right)^{\prime}-\mu^{2} V_{\phi^{3}}\left(\cos f_{i}(x)\right) \sin f_{i}(x)\right] \\
& -\frac{1}{2}\left[\left(f_{i}^{\prime}(x)\right)^{2}+\mu^{2} V_{\phi^{3}}\left(\cos f_{i}(x)\right) \cos f_{i}(x)\right]\left(v^{(\perp)}\right)^{2} \\
& -\frac{\mu^{2}}{2}\left[V_{\phi^{3}}\left(\cos f_{i}(x)\right) \cos f_{i}(x)-V_{\phi^{3} \phi^{3}}\left(\cos f_{i}(x)\right) \sin ^{2} f_{i}(x)\right]\left(v^{(\|)}\right)^{2}
\end{aligned}
$$

where $V_{\phi^{3}}$ is the derivative of $V$ with respect to $\phi^{3}$. Defining the linearized conjugate momenta

$$
\begin{aligned}
\pi_{(\perp)} & :=\frac{\partial \tilde{\mathcal{L}}}{\partial \dot{v}^{(\perp)}}=\lambda_{1} k_{i}(x) \dot{v}^{(\perp)}, \quad k_{i}(x):=1+\nu^{2}\left(f_{i}^{\prime}(x)\right)^{2} \\
\pi_{(\|)} & :=\frac{\partial \tilde{\mathcal{L}}}{\partial \dot{v}^{(\|)}}=\lambda_{1} \dot{v}^{(\|)}
\end{aligned}
$$

it is now easy to write down the linearized Hamiltonian in terms of (parameter) fields and conjugate momenta $\vec{\pi}=\left(\pi_{(\|)}, \pi_{(\perp)}\right)$, i.e.

$$
\begin{aligned}
\tilde{\mathcal{H}}_{i}\left(\vec{v}, \vec{v}^{\prime}, \vec{\pi}\right) & =\vec{\pi} \cdot \dot{\vec{v}}-\tilde{\mathcal{L}}_{i}\left(\vec{v}, \vec{v}^{\prime}, \dot{\vec{v}}\right) \\
& =\lambda_{1}\left\{\frac{1}{2} \frac{\left(\pi_{(\perp)}\right)^{2}}{k_{i}(x)}+\frac{1}{2}\left(\pi_{(\|)}\right)^{2}+\mathcal{U}_{i}\left(u, v, u^{\prime}, v^{\prime}\right)\right\}
\end{aligned}
$$

To insert (43) into (27), we also have to take care of the change in the integration measure. We are performing a substitution $\vec{\phi}(\tau, x) \mapsto \vec{v}(\tau, x)$. Since we now use parameter fields which respect the constraint $|\vec{\phi}|=1$, we get rid of the delta distribution in the integral. From the general theory of distributions, it is well-known 
that for the parametrization $\vec{\phi}=\vec{\phi}(\vec{f})$,

$$
\begin{aligned}
\prod_{i=1}^{3}\left[\mathrm{~d} \phi^{i}(\tau, x)\right] \delta(|\vec{\phi}|-1) & =\left\|\frac{\partial \vec{\phi}}{\partial f^{(\|)}} \wedge \frac{\partial \vec{\phi}}{\partial f^{(\perp)}}\right\|\left[\mathrm{d} f^{(\|)}(\tau, x)\right]\left[\mathrm{d} f^{(\perp)}(\tau, x)\right] \\
& =\left(1+\left(f^{(\perp)}(\tau, x)^{2}\right)\right)^{-\frac{3}{2}}\left[\mathrm{~d} f^{(\|)}(\tau, x)\right]\left[\mathrm{d} f^{(\perp)}(\tau, x)\right]
\end{aligned}
$$

The change to the fluctuations as integration variables is simply a shift, $f^{(\|)}(\tau, x) \mapsto$ $v^{(\|)}(\tau, x)=f^{(\|)}(\tau, x)-f_{i}(x), f^{(\perp)}(\tau, x) \mapsto v^{(\perp)}(\tau, x)=f^{(\perp)}(\tau, x)$, and we have

$$
\begin{aligned}
& \left(1+\left(f^{(\perp)}(\tau, x)\right)^{2}\right)^{-\frac{3}{2}}\left[d f^{(\|)}(\tau, x)\right]\left[d f^{(\perp)}(\tau, x)\right] \\
= & \left(1+\left(v^{(\perp)}(\tau, x)\right)^{2}\right)^{-\frac{3}{2}}\left[d v^{(\perp)}(\tau, x)\right]\left[d v^{(\|)}(\tau, x)\right] \\
\approx & {\left[d v^{(\perp)}(\tau, x)\right]\left[d v^{(\|)}(\tau, x)\right], }
\end{aligned}
$$

neglecting corrections of order $\mathcal{O}\left(\left(v^{(\perp)}(\tau, x)\right)^{2}\right)$. We also neglect corrections from the substitution $\vec{\Pi}(\tau, x) \mapsto \vec{\pi}(\tau, x)$ which is a more subtle point.

Therefore, we are now left with two contributions to the partition function, given by the integrals $(i=0,1)$

$$
\begin{gathered}
\mathcal{Z}_{i} \approx \int\left[d v^{(\perp)}(\tau, x)\right]\left[d v^{(\|)}(\tau, x)\right]\left[d \pi_{(\perp)}(\tau, x)\right]\left[d \pi_{(\|)}(\tau, x)\right] \\
\times e^{-\int_{0}^{\beta_{T}} d \tau \int_{-\infty}^{\infty} d x\left(-i \vec{\pi} \overrightarrow{\vec{v}}+\tilde{\mathcal{H}}_{i}\left(\vec{v}, \vec{v}^{\prime}, \vec{\pi}\right)\right)} .
\end{gathered}
$$

The Hamiltonian in (46) is of standard type (29) with an extra factor to the mass $M=\lambda_{1}$ (which reduces to 1 for the vacuum solution $\vec{\varphi}_{0}$ given by $f_{0}(x)=1$ ). Thus we may perform the standard momentum integration (30) which yields

$$
\mathcal{Z}_{i} \approx \int \mathcal{D}\left\{\sqrt{k_{i}(x)} v^{(\perp)}(\tau, x)\right\} \int \mathcal{D}\left\{v^{(\|)}(\tau, x)\right\} e^{-\tilde{S}_{e, i}[\vec{v}]}
$$

where the Euclidean linearized action is given by (with partial integrations)

$$
\begin{aligned}
\tilde{S}_{e, i}[\vec{v}]= & \int_{0}^{\beta_{T}} d \tau \int_{-\infty}^{\infty} d x \tilde{\mathcal{L}}_{e, i}\left(\vec{v}, \vec{v}^{\prime}, \dot{\vec{v}}\right) \\
= & \lambda_{1} \int_{0}^{\beta_{T}} d \tau \int_{-\infty}^{\infty} d x\left(\frac{1}{2}\left[k_{i}(x)\left(\dot{v}^{(\perp)}\right)^{2}+\left(\dot{v}^{(\|)}\right)^{2}\right]+\mathcal{U}_{i}\left(\vec{v}, \vec{v}^{\prime}\right)\right) \\
= & S_{e}\left[\vec{\varphi}_{i}\right] \\
& -\lambda_{1} \int_{0}^{\beta_{T}} d \tau \int_{-\infty}^{\infty} d x v^{(\|)}(\tau, x)\left[f_{i}^{\prime \prime}(x)+\mu^{2} V_{\phi^{3}}\left(\cos f_{i}(x)\right) \sin f_{i}(x)\right] \\
& +\frac{1}{2} \lambda_{1} \int_{0}^{\beta_{T}} d \tau \int_{-\infty}^{\infty} d x v^{(\|)}(\tau, x) \hat{\mathcal{G}}_{\|}^{(i)} v^{(\|)}(\tau, x) \\
& +\frac{1}{2} \lambda_{1} \int_{0}^{\beta_{T}} d \tau \int_{-\infty}^{\infty} d x v^{(\perp)}(\tau, x) \hat{\mathcal{G}}_{\perp}^{(i)} v^{(\perp)}(\tau, x) .
\end{aligned}
$$


For the vacuum we have $S_{e}\left[\vec{\varphi}_{0}\right]=0$, and

$$
S_{e}\left[\vec{\varphi}_{1}\right]=\beta_{T} \lambda_{1} \int\left[\frac{1}{2}\left(f^{\prime}(x)\right)^{2}+\mu^{2} V(\cos f(x))\right] d x
$$

is the Euclidean action of the (static!) sphaleron which obviously equals its (static) energy (4) times $\beta_{T}, S_{e}\left[\vec{\varphi}_{1}\right]=S_{s p h}\left(\beta_{T}\right)=\beta_{T} \mathcal{E}\left[\vec{\varphi}_{1}\right]=\beta_{T} \mathcal{E}_{s p h}$.

The part linear in the fluctuations, (49), vanishes if

$$
\begin{aligned}
f_{i}^{\prime \prime}(x)-\mu^{2} V_{\phi^{3}}\left(\cos f_{i}(x)\right) \sin f_{i}(x) & =0 \\
\Rightarrow \quad & f_{i}^{\prime \prime}(x)-\mu^{2} \sin f_{i}(x)=0, \quad \mu^{2}:=\frac{\lambda_{0}}{\lambda_{1}}
\end{aligned}
$$

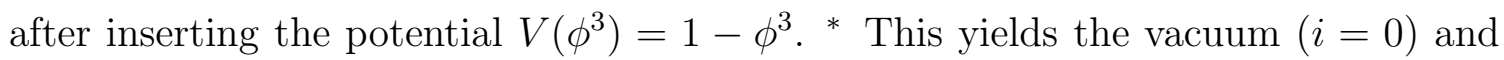
the well-known classical static solution $(i=1)(20)$.

The quadratic contributions (50) are written in terms of the (full, Euclidean) Gaussian fluctuation operators

$$
\begin{aligned}
\hat{\mathcal{G}}_{\|}^{(i)} & =-\frac{\partial^{2}}{\partial \tau^{2}}-\frac{\partial^{2}}{\partial x^{2}}-\mu^{2}\left\{V_{\phi^{3}}\left(\cos f_{i}(x)\right) \cos f_{i}(x)-V_{\phi^{3} \phi^{3}}\left(\cos f_{i}(x)\right) \sin ^{2} f_{i}(x)\right\} \\
& =-\frac{\partial^{2}}{\partial \tau^{2}}-\frac{\partial^{2}}{\partial x^{2}}+\mu^{2} \cos f_{i}(x) \\
\hat{\mathcal{G}}_{\perp}^{(i)} & =-\left\{1+\nu^{2}\left(f_{i}^{\prime}(x)\right)^{2}\right\} \frac{\partial^{2}}{\partial \tau^{2}}-\frac{\partial^{2}}{\partial x^{2}}-\left\{\mu^{2} V_{\phi^{3}}\left(\cos f_{i}(x)\right) \cos f_{i}(x)+\left(f_{i}^{\prime}(x)\right)^{2}\right\} \\
& =-\left\{1+\nu^{2}\left(f_{i}^{\prime}(x)\right)^{2}\right\} \frac{\partial^{2}}{\partial \tau^{2}}-\frac{\partial^{2}}{\partial x^{2}}+\mu^{2}\left(3 \cos f_{i}(x)-2\right)
\end{aligned}
$$

where we inserted $V\left(\phi^{3}\right)=1-\phi^{3}$. These operators describe fluctuations parallel and perpendicular to the solutions $\vec{\varphi}_{i}$.

Writing the definition of operator determinants [28]

$$
\begin{aligned}
\int \mathcal{D}\left\{v^{(\|)}(\tau, x)\right\} e^{-\frac{1}{2} \lambda_{1} \int_{0}^{\beta_{T}} d \tau \int_{-\infty}^{\infty} d x v^{(\|)}(\tau, x) \hat{\mathcal{G}}_{\|}^{(i)} v^{(\|)}(\tau, x)} & =\frac{1}{\sqrt{\operatorname{det}\left(\hat{\mathcal{G}}_{\|}^{(i)}\right)}},(56 \\
\int \mathcal{D}\left\{\sqrt{k_{i}(x)} v^{(\perp)}(\tau, x)\right\} e^{-\frac{1}{2} \lambda_{1} \int_{0}^{\beta_{T}} d \tau \int_{-\infty}^{\infty} d x v^{(\perp)}(\tau, x) \hat{\mathcal{G}}_{\perp}^{(i)} v^{(\perp)}(\tau, x)} & =\frac{1}{\sqrt{\operatorname{det}\left(\hat{\mathcal{G}}_{\perp}^{(i)}\right)}}(57
\end{aligned}
$$

(the "mass" $M=\lambda_{1}$ is absorbed in the path integration with measure $\mathcal{D}\{\ldots\}$ depending on $\lambda_{1}$, and we will see why the measure factor $\sqrt{k_{i}(x)}$ in (57) is necessary

\footnotetext{
* Eq. (52) can also be solved for other potentials of the class $V\left(\phi^{3}\right)=\left(1-\phi^{3}\right)^{k}, k \in \mathbb{N}$. For $k=2$, e.g., one obtains $f(x)=2 \operatorname{arccot}(\sqrt{2} \mu x)$.

Also, the fluctuation equations for these potentials can be obtained easily from the general form of the equations given in the text. But already for $k=2$, this yields eigenvalue equations to which no explicit solutions are known.
} 
for some similar reasons) yields in the one-loop approximation

$$
\mathcal{Z}_{i}=\frac{e^{-S\left[\vec{\varphi}_{i}\right]}}{\sqrt{\operatorname{det}\left(\hat{\mathcal{G}}_{\|}^{(i)}\right) \operatorname{det}\left(\hat{\mathcal{G}}_{\perp}^{(i)}\right)}} .
$$

Inserting these results into (26) finally leads to the following general formula for the transition rate:

$$
\Gamma=-\frac{|\varrho|}{\pi} e^{-\beta_{T} \mathcal{E}\left[\vec{\varphi}_{1}\right]} \operatorname{Im} \sqrt{\operatorname{det}\left(\frac{\left(\hat{\mathcal{G}}_{\|}^{(0)}\right.}{\left(\hat{\mathcal{G}}_{\|}^{(1)}\right.}\right) \operatorname{det}\left(\frac{\hat{\mathcal{G}}_{\perp}^{(0)}}{\hat{\mathcal{G}}_{\perp}^{(1)}}\right)}
$$

We therefore have to evaluate the determinants of the Euclidean Gaussian fluctuation operators, using appropriate regularisation techniques.

\section{Fluctuations around the vacuum}

We first consider the vacuum contribution $\mathcal{Z}_{0}$, i. e. $i=0$ in all formulae of section 3. This yields $S_{e}\left[\vec{\varphi}_{0}\right]=0$ and the measure factor $k_{0}(x)=1$, and the fluctuation operators are simply

$$
\hat{\mathcal{G}}_{\perp}^{(0)}=\hat{\mathcal{G}}_{\|}^{(0)}=\hat{\mathcal{G}}^{(0)}=-\frac{\partial^{2}}{\partial \tau^{2}}-\frac{\partial^{2}}{\partial x^{2}}+\mu^{2}=-\frac{\partial^{2}}{\partial \tau^{2}}+\mu\left[\frac{\partial^{2}}{\partial y^{2}}+1\right]
$$

with $y=\mu x, \mu>0$. $\hat{\mathcal{G}}^{(0)}$ has only a continuous spectrum given by

$$
\left(\frac{2 \pi n}{\beta_{T}}\right)^{2}+\mu^{2}\left(1+k^{2}\right), \quad k \in \mathbb{R},
$$

the corresponding generalised eigenfunctions (which are not square integrable) being $\Psi_{n, k}(\tau, x)=e^{\frac{2 \pi i n}{\beta} T} \psi_{k}(\mu x)$ with $\psi_{k}(y)=e^{i k y}$.

Decomposing the fluctuations $v^{(\|)}, v^{(\perp)}$ in terms of the eigenfunctions $\Psi_{n, k}(\tau, x)$ (which is commonly called "Fourier-Matsubara decomposition" [13]) thus yields

$$
\int \mathcal{D}\{v(\tau, x)\} e^{-\frac{1}{2} \lambda_{1} \int_{0}^{\beta_{T}} d \tau \int_{-\infty}^{\infty} d x v(\tau, x) \hat{\mathcal{G}}^{(0)} v(\tau, x)}=\prod_{k \in \mathbb{R}} \prod_{n=-\infty}^{\infty}\left\{\left(\frac{2 \pi n}{\beta_{T}}\right)^{2}+\mu^{2}\left(1+k^{2}\right)\right\}^{-\frac{1}{2}}
$$

$v=v^{(\|)}, v^{(\perp)}$. This infinite product has to be regularised in a convenient way; usually this is done by zeta function techniques [28]. For fixed $k$, one can perform the $n=-\infty \ldots \infty$-product which yields the partition function of an harmonic oscillator with frequency $\omega(k):=\mu \sqrt{1+k^{2}}$,

$$
\begin{aligned}
& \mathcal{Z}(\omega(k)):=\frac{1}{2 \sinh \frac{\omega(k) \beta_{T}}{2}}=\exp \left(-\beta_{T} \Phi\left(k^{2} ; \beta_{T}, \mu\right)\right), \\
& \Phi\left(k^{2} ; \beta_{T}, \mu\right):=\frac{\mu}{2} \sqrt{1+k^{2}}+\frac{1}{\beta_{T}} \ln \left(1-e^{-\beta_{T} \mu \sqrt{1+k^{2}}}\right) .
\end{aligned}
$$


The notation $\Phi\left(k^{2} ; \beta_{T}, \mu\right)=\Phi_{1}\left(k^{2} ; \mu\right)+\frac{1}{\beta_{T}} \Phi_{2}\left(k^{2} ; \beta_{T} \mu\right)$ with

$$
\Phi_{1}\left(k^{2} ; \mu\right)=\frac{\mu}{2} \sqrt{1+k^{2}}, \quad \Phi_{2}\left(k^{2} ; a\right)=\ln \left(1-e^{-a \sqrt{1+k^{2}}}\right)
$$

will be useful in the following.

What remains to be done is the product over all $k \in \mathbb{R}$. Taking the logarithm of (62), we write it as an integral over $k$ weighted with the density of the continuous vacuum fluctuation states $\rho_{0}$ (which will be discussed later). The final result for the vacuum determinants is thus

$$
\frac{1}{\sqrt{\operatorname{det}\left(\hat{\mathcal{G}}_{\|}^{(0)}\right)}}=\frac{1}{\sqrt{\operatorname{det}\left(\hat{\mathcal{G}}_{\perp}^{(0)}\right)}}=\exp \left(-\beta_{T} \int_{-\infty}^{\infty} d k \rho_{0}(k) \Phi\left(k^{2} ; \beta_{T}, \mu^{2}\right)\right)
$$

\section{Parallel fluctuation contributions to the transi- tion rate}

The determinant of $\hat{\mathcal{G}}_{\|}^{(1)}$ can also be calculated with a standard Fourier-Matsubara decomposition, i.e.

$$
\begin{aligned}
\hat{\mathcal{G}}_{\|}^{(1)} & =-\frac{\partial^{2}}{\partial \tau^{2}}-\frac{\partial^{2}}{\partial x^{2}}+\mu^{2}\left\{1-2 \operatorname{sech}^{2}(\mu x)\right\} \\
& =-\frac{\partial^{2}}{\partial \tau^{2}}+\hat{\mathcal{H}}_{\|}
\end{aligned}
$$

has eigenfunctions $\Psi_{n m}^{(\|)}(\tau, x)=e^{\frac{2 \pi i n}{\beta} T} \psi_{m}^{(\|)}(x)$ with eigenvalues

$$
\left(\frac{2 \pi n}{\beta_{T}}\right)^{2}+\left(\omega_{m}^{(\|)}\right)^{2}
$$

where $\left(\omega_{m}^{(\|)}\right)^{2}$ are the eigenvalues of

$$
\hat{\mathcal{H}}_{\|} \psi_{m}^{(\|)}=-\frac{\partial^{2} \psi_{m}^{(\|)}}{\partial x^{2}}+\mu^{2}\left\{1-2 \operatorname{sech}^{2}(\mu x)\right\} \psi_{m}^{(\|)}=\left(\omega_{m}^{(\|)}\right)^{2} \psi_{m}^{(\|)}
$$

which is just the static fluctuation equation (24). This is a special case of eq. (170) discussed in Appendix $\mathbb{B}$ with $U=s(s+1) \equiv 2, s=s_{\|}=1$ and $E=$ $\frac{1}{\mu^{2}}\left(\omega_{m}^{(\|)}\right)^{2}-1$ which is known as Pöschl-Teller equation 42. It has a normalised zero mode $\psi_{0}^{(\|)}(x)=\sqrt{\frac{\mu}{2}} \operatorname{sech}(\mu x),\left(\omega_{0}^{(\|)}\right)^{2}=0$, and a continuum $\left(\omega^{(\|)}\right)^{2}(k)=\mu^{2}(1+$ $\left.k^{2}\right)$. Decomposing the parallel fluctuations $v^{(\|)}$into eigenstates of $\hat{\mathcal{G}}_{\|}^{(1)}, v^{(\|)}(\tau, x)=$ $\frac{1}{\sqrt{\beta_{T}}} \sum_{n, m} c_{n m}^{(\|)} \Psi_{n m}^{(\|)}(\tau, x)$, yields

$$
\begin{gathered}
\int \mathcal{D}\left\{v^{(\|)}(\tau, x)\right\} e^{-\frac{\lambda 1}{2} \int_{0}^{\beta_{T}} d \tau \int_{-\infty}^{\infty} d x v^{(\|)}(\tau, x) \hat{\mathcal{G}}_{\|}^{(1)} v^{(\|)}(\tau, x)} \\
=\prod_{m} \prod_{n=-\infty}^{\infty}\left\{\left(\frac{2 \pi n}{\beta_{T}}\right)^{2}+\left(\omega_{m}^{(\|)}\right)^{2}\right\}^{-\frac{1}{2}}
\end{gathered}
$$


where $m$ now runs over the values $m=0$ and $m=k, k \in \mathbb{R}$. Again (assuming zeta function regularisation), the $n$-product yields one harmonic oscillator for each fixed $m$.

To illustrate this appearence of harmonic oscillators, one can also decompose the parallel fluctuations in terms of the eigenfunctions of eq. (69) which can be normalized with respect to the scalar product

$$
\left\langle\xi_{1}, \xi_{2}\right\rangle_{\|}:=\int_{-\infty}^{\infty} \xi_{1}^{*}(x) \xi_{2}(x) d x
$$

on $L^{2}(\mathbb{R}):\left\langle\psi_{n}^{(\|)}, \psi_{m}^{(\|)}\right\rangle_{\|}=\delta_{n m}$ (with the usual generalisation for scattering states which we assume throughout in the following). This decomposition

$$
v^{(\|)}(\tau, x)=\sum_{m} c_{m}^{(\|)}(\tau) \psi_{m}^{(\|)}(x)
$$

is related to the Fourier-Matsubara decomposition used above by

$$
c_{m}^{(\|)}(\tau)=\frac{1}{\sqrt{\beta_{T}}} \sum_{n=-\infty}^{\infty} c_{n m}^{(\|)} e^{\frac{2 \pi i n}{\beta_{T}} \tau} .
$$

For fixed $\tau$, (72) defines a mapping $L^{2}(\mathbb{R}) \rightarrow \ell$ which is an isometry with respect to the $L^{2}(\mathbb{R})$ scalar product $\langle,\rangle_{\|}$. As this scalar product corresponds to the measure in the fluctuation path integral (70), we may use (72) as substitution in the integral without unit Jacobian. This yields

$$
\begin{aligned}
& \int \mathcal{D}\left\{v^{(\|)}(\tau, x)\right\} e^{-\frac{1}{2} \int_{0}^{\beta} T d \tau \int_{-\infty}^{\infty} d x v^{(\|)}(\tau, x) \hat{\mathcal{G}}_{\|}^{(1)} v^{(\|)}(\tau, x)} \\
= & \prod_{m} \int \mathcal{D}\left\{c_{m}^{(\|)}(\tau)\right\} e^{-\frac{1}{2} \int_{0}^{\beta_{T}} d \tau\left(\left|\dot{c}_{m}^{(\|)}(\tau)\right|^{2}+\left(\omega_{m}^{(\|)}\right)^{2}\left|c_{m}^{(\|)}(\tau)\right|^{2}\right)} \\
= & \prod_{m} \frac{1}{2 \sinh \frac{\omega_{m}^{(\|)} \beta_{T}}{2}}
\end{aligned}
$$

Each $c_{m}^{(\|)}$-integral thus contributes one harmonic oscillator with frequency $\omega_{m}^{(\|)}$. We will use this decomposition technique to evaluate the perpendicular fluctuation integral (57) where the Fourier-Matsubara decomposition of the fluctuations is not so obvious. Details of the substitution used in (74) can therefore be found in section 6 .

In (70), the only difference compared with the vacuum calculation is the zero eigenvalue $m=0$ of $\mathcal{H}_{\|}$. This yields a zero mode factor 29 in the determinant of the parallel sphaleron fluctuations which is the partition function of an harmonic oscillator with zero frequency, i. e. that of a free mode which has to be treated separately. This zero mode factor originates from the translation symmetry of the model which is broken by the classical solution $\vec{\varphi}(x)$ which is discussed in Appendix 
A. The result of this (formal) evaluation of the $n$-product in (70) for $m=0$ is thus the replacement

$$
\prod_{n=-\infty}^{\infty}\left\{\left(\frac{2 \pi n}{\beta_{T}}\right)^{2}+\left(\omega_{0}^{(\|)}\right)^{2}\right\}^{-\frac{1}{2}}=\frac{1}{2 \sinh \frac{\omega_{0}(\|) \beta_{T}}{2}} \longrightarrow L \mathcal{N}_{\|}:=2 L \sqrt{\lambda_{1}} \sqrt{\frac{\mu}{\pi \beta_{T}}}
$$

where we introduced a finite space length $L$ to get rid of the volume divergence. (Later we will divide the transition rate by $L$ to obtain the transition rate per unit volume which is independent of this procedure.)

The continuous product over $k \in \mathbb{R}$ is performed in exactly the same way as in Section 1 and yields

$$
\prod_{k \in \mathbb{R}} \frac{1}{2 \sinh \frac{\omega^{(\|)}(k) \beta_{T}}{2}}=\exp \left(-\beta_{T} \int_{-\infty}^{\infty} d k \rho_{\|}(k) \Phi\left(k^{2} ; \beta_{T}, \mu\right)\right)
$$

where $\Phi\left(k^{2} ; \beta_{T} \mu\right)$ is defined in (64) and $\rho_{\|}(k)$ is the density of the continuum states of $\hat{\mathcal{H}}_{\|}$.

Together with eq. (66) we thus have

$$
\sqrt{\operatorname{det}\left(\frac{\left(\hat{\mathcal{G}}_{\|}^{(0)}\right.}{\left(\hat{\mathcal{G}}_{\|}^{(1)}\right.}\right)}=L \mathcal{N}_{\|} \exp \left(-\beta_{T} \int_{-\infty}^{\infty} d k\left(\rho_{\|}(k)-\rho_{0}(k)\right) \Phi\left(k^{2} ; \beta_{T}, \mu\right)\right) \text {. }
$$

This "parallel fluctuation contribution" to the transition rate (59) is a real quantity; we thus expect the perpendicular contribution to be imaginary.

The integral in (77), containing the difference of the densities of continuum states of two Hamiltonians, can be evaluated using standard techniques discussed in refs. [2, 19, 20, 30, 31, 32]. The main formula is

$$
\int_{-\infty}^{\infty} d k\left(\rho_{\|}(k)-\rho_{0}(k)\right) \Phi\left(k^{2} ; \beta_{T}, \mu\right)=\frac{1}{2 \pi} \int_{-\infty}^{\infty} d k \Phi\left(k^{2} ; \beta_{T}, \mu\right) \frac{d}{d k} \delta_{\|}(k)
$$

where $\delta_{\|}(k)$ is the phase shift between incoming and outgoing asymptotic plane waves in the scattering states of eq. (69) as discussed in Appendix B, eq. (197): $\delta_{\|}(k)=$ $\delta_{1}(k)=-2 \arctan (k)$ since $s=s_{\|}=1$.

Inserting (78) into the integral of (77) yields for the continuum fluctuation contributions

$$
\int_{-\infty}^{\infty} d k\left(\rho(k)-\rho_{0}(k)\right) \Phi\left(k^{2} ; \beta_{T}, \mu\right)=-\frac{\mu}{2 \pi} \int_{-\infty}^{\infty} d k \frac{d k}{\sqrt{1+k^{2}}}+\frac{1}{\beta_{T}} h_{\|}\left(\beta_{T} \mu\right)
$$

The first integral in (79) is logarithmically divergent; it is the sum of zero-point energy contributions of the oscillators in the Fourier-Matsubara decomposition (70) and has to be renormalised. We ignore this here. 
The function $h_{\|}$is given by

$$
\begin{aligned}
h_{\|}(a) & =\frac{1}{2 \pi} \int_{-\infty}^{\infty} d k \Phi_{2}(k ; a) \frac{d \delta_{\|}(k)}{d k} \\
& =-\frac{1}{\pi} \int_{-\infty}^{\infty} d k \frac{\ln \left(1-e^{-a \sqrt{1+k^{2}}}\right)}{1+k^{2}}>0 .
\end{aligned}
$$

The integral is convergent for all $a>0$, and

$$
h_{\|}\left(\beta_{T} \mu\right) \sim-\ln \left(\beta_{T} \mu\right)>0
$$

in the high temperature limit $\beta_{T} \rightarrow 0\left(a=\beta_{T} \mu \rightarrow 0\right)$ which is shown in Appendix Q, eq. (203).

Finally, collecting all results, the parallel fluctuation contribution to the transition rate $(77)$ reads

$$
\sqrt{\operatorname{det}\left(\frac{\left(\hat{\mathcal{G}}_{\|}^{(0)}\right.}{\left(\hat{\mathcal{G}}_{\|}^{(1)}\right.}\right)}=L \mathcal{N}_{\|} e^{-h_{\|}\left(\beta_{T} \mu\right)}
$$

\section{Perpendicular fluctuation contributions to the transition rate}

In order to calculate the remaining perpendicular fluctuation contribution to the transition rate (59), we have to evaluate the perpendicular fluctuation integral (57) $(i=1)$ around the sphaleron. Here the measure factor (41),

$$
k_{1}(x) \equiv k(x):=1+\nu^{2}\left(f^{\prime}(x)\right)^{2}=1+4 \kappa^{2} \operatorname{sech}^{2}(\mu x)
$$

with $\kappa^{2}=\mu^{2} \nu^{2}$ is the important new ingredient which contains the influence of the Skyrme term on the model. The fluctuation integral reads

$$
\int \mathcal{D}\left\{\sqrt{k(x)} v^{(\perp)}(\tau, x)\right\} e^{-\frac{1}{2} \lambda_{1} \int_{0}^{\beta} T d \tau \int_{-\infty}^{\infty} d x v^{(\perp)}(\tau, x) \hat{\mathcal{G}}_{\perp}^{(1)} v^{(\perp)}(\tau, x)}
$$

with

$$
\hat{\mathcal{G}}_{\perp}=-k(x) \frac{\partial^{2}}{\partial \tau^{2}}+\hat{\mathcal{H}}_{\perp}, \quad \hat{\mathcal{H}}_{\perp}=-\frac{\partial^{2}}{\partial x^{2}}+\mu^{2}\left\{1-6 \operatorname{sech}^{2}(\mu x)\right\}
$$

where $\hat{\mathcal{H}}_{\perp}$ is the static Gaussian fluctuation operator (25) of the static energy functional.

\subsection{The measure-modified harmonic oscillator decomposi- tion}

To obtain the desired Fourier-Matsubara decomposition in (84), we solve the following "measure-modified static fluctuation equation" [33]

$$
\hat{\mathcal{H}}_{\perp} \psi^{(\perp)}(x)=\left(\omega^{(\perp)}\right)^{2} k(x) \psi^{(\perp)}(x)
$$


which is a general Sturm-Liouville problem on $L^{2}(\mathbb{R})$ with the metric factor $k(x)$ on the right hand side multiplying the eigenvalue [34]. (This equation is different from (25). We are no longer considering the sphaleron in the space of static field configurations where it was first constructed, cf. Section 2, but embedded it into the full euclidean field theory!) From Sturm-Liouville theory, we know that the eigenfunctions of (86) are orthogonal with respect to the scalar product

$$
\left\langle\xi_{1}, \xi_{2}\right\rangle_{\perp}:=\int_{-\infty}^{\infty} \xi_{1}^{*}(x) \xi_{2}(x) k(x) d x
$$

We may thus orthonormalize the eigenfunctions $\hat{\mathcal{H}}_{\perp} \psi_{m}^{(\perp)}=\left(\omega_{m}^{(\perp)}\right)^{2} k(x) \psi_{m}^{(\perp)}$ of $(86)$, $\left\langle\psi_{m}^{(\perp)}, \psi_{l}^{(\perp)}\right\rangle_{\perp}=\delta_{l m}$ and expand the fluctuations $v^{(\perp)}$ in a Fourier-series with $\tau$ dependent periodic coefficients:

$$
v^{(\perp)}(\tau, x)=\sum_{m} c_{m}^{(\perp)}(\tau) \psi_{m}^{(\perp)}(x)
$$

(This expansion again contains generalised scattering eigenfunctions in the usual sense).

The fluctuations are real, so that $\sum_{m} c_{m}^{(\perp)}(\tau) \psi_{m}^{(\perp)}(x)=\sum_{m}\left(c_{m}^{(\perp)}\right)^{*}(\tau)\left(\psi_{m}^{(\perp)}\right)^{*}(x)$. Inserting (88) into the integrand of (84) yields

$$
\begin{aligned}
& v^{(\perp)}(\tau, x) \hat{\mathcal{G}}_{\perp}^{(1)} v^{(\perp)}(\tau, x) \\
& =\left(\sum_{m}\left(c_{m}^{(\perp)}\right)^{*}(\tau)\left(\psi_{m}^{(\perp)}\right)^{*}(x)\right)\left[-k(x) \frac{\partial^{2}}{\partial \tau^{2}}+\hat{\mathcal{H}}_{\perp}\right]\left(\sum_{l} c_{l}^{(\perp)}(\tau) \psi_{l}^{(\perp)}(x)\right) \\
& =\left(\sum_{m}\left(c_{m}^{(\perp)}\right)^{*}(\tau)\left(\psi_{m}^{(\perp)}\right)^{*}(x)\right) \\
& \quad \times\left(\sum_{l}\left[-k(x) \ddot{c}_{l}^{(\perp)}(\tau) \psi_{l}^{(\perp)}(x)+\left(\omega_{l}^{(\perp)}\right)^{2} k(x) c_{l}^{(\perp)}(\tau) \psi_{l}^{(\perp)}(x)\right]\right) \\
& =\sum_{m} \sum_{l}\left[-\left(c_{m}^{(\perp)}\right)^{*}(\tau) \ddot{c}_{l}^{(\perp)}(\tau)+\left(\omega_{l}^{(\perp)}\right)^{2}\left(c_{m}^{(\perp)}\right)^{*}(\tau) c_{l}^{(\perp)}(\tau)\right] \\
& \quad \times\left[\left(\psi_{m}^{(\perp)}\right)^{*}(x) \psi_{l}^{(\perp)}(x) k(x) d x\right],
\end{aligned}
$$

thus

$$
\begin{aligned}
& \int_{0}^{\beta_{T}} d \tau \int_{-\infty}^{\infty} d x v^{(\perp)}(\tau, x) \hat{\mathcal{G}}_{\perp}^{(1)} v^{(\perp)}(\tau, x) \\
& =\sum_{m} \sum_{l}\left[\int_{0}^{\beta_{T}} d \tau\left(-\left(c_{m}^{(\perp)}\right)^{*}(\tau) \ddot{c}_{l}^{(\perp)}(\tau)+\left(\omega_{l}^{(\perp)}\right)^{2}\left(c_{m}^{(\perp)}\right)^{*}(\tau) c_{l}^{(\perp)}(\tau)\right)\right] \\
& \quad \times \underbrace{\left\langle\psi_{m}^{(\perp)}, \psi_{l}^{(\perp)}\right\rangle_{\perp}}_{=\delta_{l m}} \\
& =\sum_{m} \int_{0}^{\beta_{T}} d \tau\left(-\left(c_{m}^{(\perp)}\right)^{*}(\tau) \ddot{c}_{m}^{(\perp)}(\tau)+\left(\omega_{m}^{(\perp)}\right)^{2}\left(c_{m}^{(\perp)}\right)^{*}(\tau) c_{m}^{(\perp)}(\tau)\right)
\end{aligned}
$$




$$
=\sum_{m} \int_{0}^{\beta_{T}} d \tau\left(\left|\dot{c}_{m}^{(\perp)}(\tau)\right|^{2}+\left(\omega_{m}^{(\perp)}\right)^{2}\left|c_{m}^{(\perp)}(\tau)\right|^{2}\right)
$$

This is a sum over harmonic oscillators. In particular, there is no contribution from the measure factor $k$ in this sum (it disappears with the help of Sturm-Liouville theory).

The mapping $v^{(\perp)} \mapsto c_{m}^{(\perp)}$ for fixed $\tau$ is an isometry $L^{2}(\mathbb{R}) \rightarrow \ell^{2}$ with respect to the scalar product $\langle,\rangle_{\perp}$ containing the measure factor $k(x)$ which also appears in the path integral measure, so we may substitute (88) into (84) with unit Jacobian:

$$
\begin{aligned}
& \int \mathcal{D}\left\{\sqrt{k(x)} v^{(\perp)}(\tau, x)\right\} e^{-\frac{1}{2} \lambda_{1} \int_{0}^{\beta_{T}} d \tau \int_{-\infty}^{\infty} d x v^{(\perp)}(\tau, x) \hat{\mathcal{G}}_{\perp}^{(1)} v^{(\perp)}(\tau, x)} \\
& =\prod_{m} \int \mathcal{D}\left\{c_{m}^{(\perp)}(\tau)\right\} e^{-\frac{1}{2} \int_{0}^{\beta_{T}} d \tau\left(\left|\dot{c}_{m}^{(\perp)}(\tau)\right|^{2}+\left(\omega_{m}^{(\perp)}\right)^{2}\left|c_{m}^{(\perp)}(\tau)\right|^{2}\right)}
\end{aligned}
$$

Now it is easy to obtain the Fourier-Matsubara decomposition of the perpendicular fluctuations. With a Fourier expansion of the coefficients $c_{m}^{(\perp)}(\tau)$,

$$
c_{m}^{(\perp)}(\tau)=\frac{1}{\sqrt{\beta_{T}}} \sum_{n=-\infty}^{\infty} c_{n m}^{(\perp)} e^{\frac{2 \pi i n}{\beta_{T}} \tau},
$$

we have

$$
v^{(\perp)}(\tau, x)=\frac{1}{\sqrt{\beta_{T}}} \sum_{n, m} c_{n m}^{(\perp)} e^{\frac{2 \pi i n}{\beta} T} \psi_{m}^{(\perp)}(x)
$$

similar to that of the parallel fluctuations. In terms of the $c_{n m}^{(\perp)}$, the operator $\hat{\mathcal{G}}_{\perp}^{(1)}$ is completely diagonalised,

$$
\int_{0}^{\beta_{T}} d \tau \int_{-\infty}^{\infty} d x v^{(\perp)}(\tau, x) \hat{\mathcal{G}}_{\perp}^{(1)} v^{(\perp)}(\tau, x)=\sum_{n, m} c_{n m}^{(\perp)}\left[\left(\frac{2 \pi n}{\beta_{T}}\right)^{2}+\left(\omega_{m}^{(\perp)}\right)^{2}\right]\left(c_{n m}^{(\perp)}\right)^{*}
$$

and the substitution $v^{(\perp)}(\tau, x) \mapsto c_{n m}^{(\perp)}$ is again an isometry with respect to the path integral measure, thus

$$
\begin{aligned}
& \int \mathcal{D}\left\{\sqrt{k(x)} v^{(\perp)}(\tau, x)\right\} e^{-\frac{1}{2} \lambda_{1} \int_{0}^{\beta_{T}} d \tau \int_{-\infty}^{\infty} d x v^{(\perp)}(\tau, x) \hat{\mathcal{G}}_{\perp}^{(1)} v^{(\perp)}(\tau, x)} \\
& =\int \prod_{m} \prod_{n=-\infty}^{\infty} \mathcal{D}\left\{c_{n m}^{(\perp)}\right\} e^{-\frac{1}{2} \sum_{n, m} c_{n m}^{(\perp)}\left[\left(\frac{2 \pi n}{\beta_{T}}\right)^{2}+\left(\omega_{m}^{(\perp)}\right)^{2}\right]\left(c_{n m}^{(\perp)}\right)^{*}} \\
& =\prod_{m} \prod_{n=-\infty}^{\infty}\left\{\left(\frac{2 \pi n}{\beta_{T}}\right)^{2}+\left(\omega_{m}^{(\perp)}\right)^{2}\right\}^{-\frac{1}{2}} .
\end{aligned}
$$

with unit Jacobian. 
Assuming zeta function regularisation [28], we first perform the $n$-product to obtain

$$
\int \mathcal{D}\left\{\sqrt{k(x)} v^{(\perp)}(\tau, x)\right\} e^{-\frac{1}{2} \lambda_{1} \int_{0}^{\beta_{T}} d \tau \int_{-\infty}^{\infty} d x v^{(\perp)}(\tau, x) \hat{\mathcal{G}}_{\perp}^{(1)} v^{(\perp)}(\tau, x)}=\prod_{m} \frac{1}{2 \sinh \frac{\omega_{m}^{(\perp)} \beta_{T}}{2}} .
$$

This result is also clear from (91), but to controll the necessary renormalisation steps (which is not our aim here), one has to return to the Fourier-Matsubara decomposition.

\subsection{Solution and discussion of the measure-modified static fluctuation equation}

In order to use the harmonic oscillator decompostion (96), we have to solve (86). This Sturm-Liouville equation may be rewritten as (with $y=\mu x$ )

$$
\left\{\frac{d^{2}}{d y^{2}}+\left[\frac{\left(\omega^{(\perp)}\right)^{2}}{\mu^{2}}-1\right]+\left[6+4 \nu^{2}\left(\omega^{(\perp)}\right)^{2}\right] \operatorname{sech}^{2} y\right\} \psi^{(\perp)}(y)=0 .
$$

which is eq. (170) with

$$
\begin{aligned}
& E=E\left(\left(\omega^{(\perp)}\right)^{2}\right)=\frac{\left(\omega^{(\perp)}\right)^{2}}{\mu^{2}}-1 \Longrightarrow \epsilon=\sqrt{1-\frac{\left(\omega^{(\perp)}\right)^{2}}{\mu^{2}}} \\
& U=U\left(\left(\omega^{(\perp)}\right)^{2}\right)=6+4 \nu^{2}\left(\omega^{(\perp)}\right)^{2} \Longrightarrow s=\sqrt{\frac{25}{4}+4 \nu^{2}\left(\omega^{(\perp)}\right)^{2}}-\frac{1}{2} .
\end{aligned}
$$

It is convenient to discuss the results in terms of $\tilde{\omega}^{2}:=\frac{1}{\mu^{2}}\left(\omega^{(\perp)}\right)^{2}$ where the constant $\mu^{2}=\frac{\lambda_{0}}{\lambda_{1}}$ does not depend on the Skyrme coupling constant $\lambda_{2}$, so that it is possible to discuss the Skyrme-less limit $\lambda_{2} \rightarrow 0$ in terms of $\tilde{\omega}^{2}$ which will depend only on the one parameter $\kappa^{2}=\mu^{2} \nu^{2}=\frac{\lambda_{0} \lambda_{2}}{\lambda_{1}^{2}}$.

There are discrete eigenvalues $\tilde{\omega}_{m}^{2}$ for all $m \in \mathbb{N}_{0}$ with

$$
T_{U\left(\left(\omega_{m}^{(\perp)}\right)^{2}\right)}(m)=\sqrt{\frac{25}{4}+4 \kappa^{2} \tilde{\omega}_{m}^{2}}-\left(m+\frac{1}{2}\right)>0
$$

and $U\left(\left(\omega^{(\perp)}\right)^{2}\right)=6+4 \kappa^{2} \tilde{\omega}^{2}>0$ which are conditions for the existence of an eigenvalue which depend on the eigenvalue itself. If a discrete eigenvalue exists, it is given by (182) which means it is a solution of

$$
-E \equiv 1-\tilde{\omega}_{m}^{2}=\left[\sqrt{\frac{25}{4}+4 \kappa^{2} \tilde{\omega}_{m}^{2}}-\left(m+\frac{1}{2}\right)\right]^{2} .
$$


We first prove that there are no solutions of (101) with $m>2$ which satisfy (100). Assume that such a solution $\tilde{\omega}_{m}^{2}(m>2)$ exists. If it is positive, $\tilde{\omega}_{m}^{2}>0$, then $1-\tilde{\omega}_{m}^{2}<1$, but the right hand side of eq. (101) may be estimated from below:

$$
\left[\sqrt{\frac{25}{4}+4 \kappa^{2} \tilde{\omega}_{m}^{2}}-\left(m+\frac{1}{2}\right)\right]^{2} \geq\left[\sqrt{\frac{25}{4}}-\left(m+\frac{1}{2}\right)\right]^{2}=[2-m]^{2} \geq 1
$$

for $m>2$. So $\tilde{\omega}_{m}^{2}$ has to be negative or zero, $\tilde{\omega}_{m}^{2}<0$, contrary to the assumption. If $\tilde{\omega}_{m}^{2}<0$ is so negative that $6+4 \kappa^{2} \tilde{\omega}^{2}<0$, it cannot be a discrete eigenvalue because then $U\left(\omega_{m}^{2}\right)<0$. Otherwise, we may estimate (100) from above:

$$
\sqrt{\frac{25}{4}+4 \kappa^{2} \tilde{\omega}_{m}^{2}}-\left(m+\frac{1}{2}\right) \leq \sqrt{\frac{25}{4}}-\left(m+\frac{1}{2}\right)=2-m<0
$$

for $m>2$. Therefore, a solution of (100) and (101) cannot exist for $m>2$.

Next, we solve (101) naively by squaring the square root (we have to check whether the solutions we obtain really solve (101)) to get a quadratic equation

$$
A\left(\kappa^{2}\right)\left(\tilde{\omega}^{2}\right)^{2}+B\left(m, \kappa^{2}\right) \tilde{\omega}^{2}+C(m)=0
$$

with

$$
\begin{aligned}
A\left(\kappa^{2}\right) & =\left(1+4 \kappa^{2}\right)^{2} \\
B\left(m, \kappa^{2}\right) & =\frac{21}{4}\left(1+4 \kappa^{2}\right)+2\left(m+\frac{1}{2}\right)^{2}\left(1-4 \kappa^{2}\right) \\
C(m) & =\left(\frac{25}{4}-\left(m+\frac{1}{2}\right)^{2}\right)^{2}-2\left(\frac{25}{4}+\left(m+\frac{1}{2}\right)^{2}\right)+1
\end{aligned}
$$

The general solution of (104) is

$$
\tilde{\omega}^{2}=\frac{-B\left(m, \kappa^{2}\right) \pm \sqrt{\Delta\left(m, \kappa^{2}\right)}}{2 A\left(\kappa^{2}\right)}
$$

where $\Delta\left(m, \kappa^{2}\right)$ is the discriminant

$$
\Delta\left(m, \kappa^{2}\right)=4\left(m+\frac{1}{2}\right)^{2}\left[25+4\left(29-4\left(m+\frac{1}{2}\right)^{2}\right) \kappa^{2}+64\left(\kappa^{2}\right)^{2}\right]
$$

It is easy to check that $\Delta\left(m, \kappa^{2}\right)$ is nonnegative if $\kappa^{2} \geq 0$ for $m=0,1,2$.

Next, we insert the solutions $(\sqrt{106})$, i. e.

$$
\begin{aligned}
\tilde{\omega}_{m, \pm}^{2}=-\left[\frac{21}{4\left(1+4 \kappa^{2}\right)}+\left(m+\frac{1}{2}\right)^{2} \frac{\left(1-4 \kappa^{2}\right)}{\left(1+4 \kappa^{2}\right)^{2}}\right] \\
\pm \frac{\left(m+\frac{1}{2}\right)}{\left(1+4 \kappa^{2}\right)^{2}} \sqrt{25+4\left(29-4\left(m+\frac{1}{2}\right)^{2}\right) \kappa^{2}+64\left(\kappa^{2}\right)^{2}}
\end{aligned}
$$


into (101) and (100) to determine the allowed signs (we already remarked that we have to check the solutions). We have

$$
\sqrt{\frac{25}{4}+4 \kappa^{2} \tilde{\omega}_{m, \pm}^{2}}=\frac{\left|8\left(m+\frac{1}{2}\right) \pm \sqrt{25+4\left(29-4\left(m+\frac{1}{2}\right)^{2}\right) \kappa^{2}+64\left(\kappa^{2}\right)^{2}}\right|}{2\left(1+4 \kappa^{2}\right)} .
$$

The modulus is always positive for the upper (plus) sign, and one can easily check that this is a solution of eq. (101) which satisfies (100) for all $\kappa^{2} \geq 0$. But for the lower (minus) sign, we have to distinguish two cases: For $0 \leq \kappa^{2}<\frac{25}{16} \frac{1}{\left(m+\frac{1}{2}\right)^{2}-1}, \tilde{\omega}_{m,-}^{2}$ does not solve (101). Otherwise, for $\kappa^{2} \geq \frac{25}{16} \frac{1}{\left(m+\frac{1}{2}\right)^{2}-1}$, it solves this equation, but the condition (100) is not fulfilled. We therefore have to reject $\tilde{\omega}_{m,-}^{2}$ as solution to (101) under the condition (100). So finally, there are three discrete eigenvalues of (97) given by

$$
\begin{gathered}
\omega_{-}^{2}\left(\kappa^{2}\right)=\tilde{\omega}_{0}^{2}\left(\kappa^{2}\right)=\frac{\left(\omega_{0}^{(\perp)}\right)^{2}}{\mu^{2}}=\frac{-\left(20 \kappa^{2}+\frac{11}{2}\right)+\frac{1}{2} \sqrt{25+112 \kappa^{2}+64\left(\kappa^{2}\right)^{2}}}{\left(1+4 \kappa^{2}\right)^{2}}<0 \\
\tilde{\omega}_{1}^{2}\left(\kappa^{2}\right)=\frac{\left(\omega_{1}^{(\perp)}\right)^{2}}{\mu^{2}}=0 \\
\omega_{+}^{2}\left(\kappa^{2}\right)=\tilde{\omega}_{2}^{2}\left(\kappa^{2}\right)=\frac{\left(\omega_{2}^{(\perp)}\right)^{2}}{\mu^{2}}=\frac{4 \kappa^{2}-\frac{23}{2}+\frac{5}{2} \sqrt{25+16 \kappa^{2}+64\left(\kappa^{2}\right)^{2}}}{\left(1+4 \kappa^{2}\right)^{2}}>0 .
\end{gathered}
$$

There is a zero eigenvalue $\left(\omega_{1}^{(\perp)}\right)^{2}=0$. In Appendix A, we show that this zero mode is due to the $U(1)$ rotation symmetry of the Euclidean action (51) which is broken by the classical sphaleron solution $\vec{\varphi}_{1}(x)$ (analogous to the translation zero mode which can be found in the spectrum of the parallel fluctuations). This effect is independent of $\kappa^{2}$, so the zero mode exists for all $\kappa^{2}$.

It is interesting to analyse the $\kappa^{2}$-dependence of the other two discrete eigenvalues which is shown in Fig. 3. For $\kappa^{2} \rightarrow 0$, we have $\tilde{\omega}_{0}^{2}\left(\kappa^{2}=0\right)=-3$ which is the Skyrmeless result $\left(\lambda_{2} \rightarrow 0\right)$. In the same limit, we have $\tilde{\omega}_{2}^{2}\left(\kappa^{2}=0\right)=1$ which explains why there are only two discrete eigenvalues in the original model [20]: Without a Skyrme term, the third eigenvalue is the lower bound of the continuum. In the limit $\kappa^{2} \rightarrow \infty$, all three eigenvalues tend to zero. This can be interpreted as the $\lambda_{1} \rightarrow 0$ limit of the model in which there is no sphaleron solution (and therefore no stability spectrum).

The corresponding eigenfunctions are given by (184) were $s$ now depends on $\left(\omega_{m}^{(\perp)}\right)^{2}$ (cf. 99). Using (109), we have

$$
s=s_{m}\left(\kappa^{2}\right):=\frac{8 m \kappa^{2}-1+\sqrt{25+4\left(29-4\left(m+\frac{1}{2}\right)^{2}\right) \kappa^{2}+64\left(\kappa^{2}\right)^{2}}}{2\left(1+4 \kappa^{2}\right)} .
$$




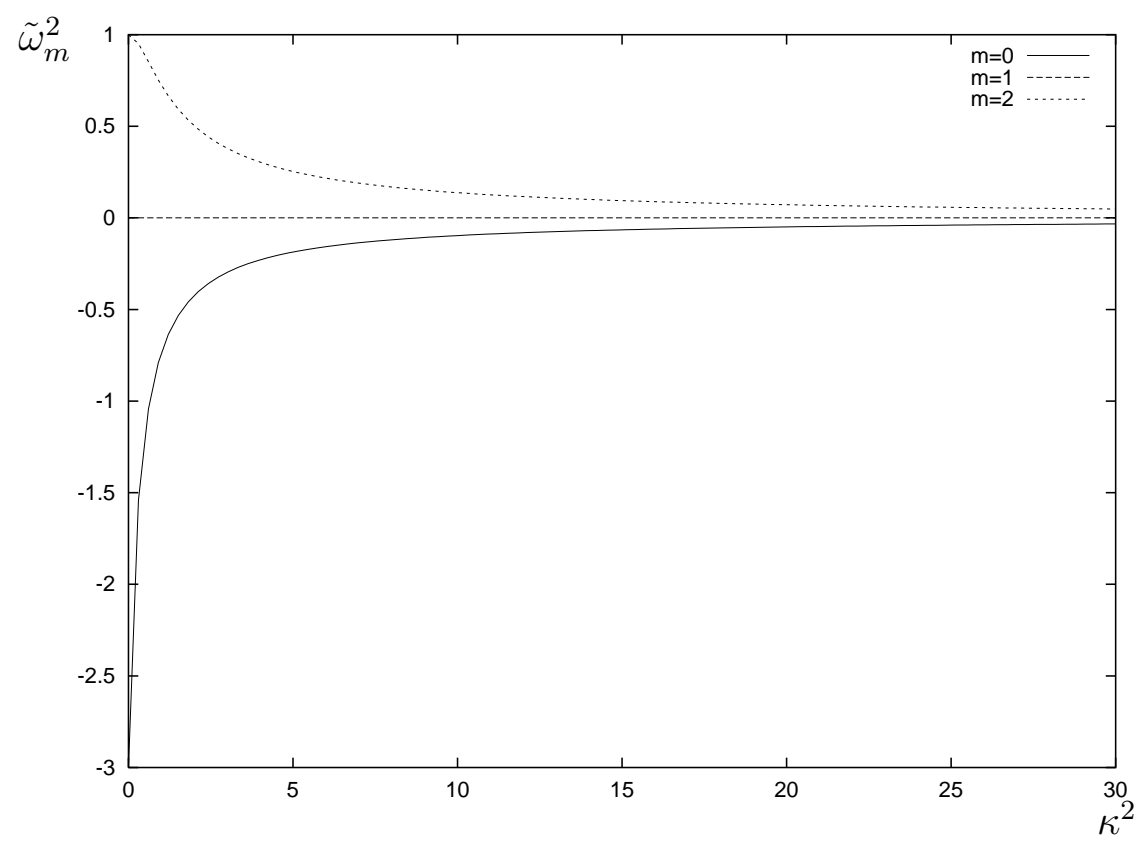

Figure 3: Discrete eigenvalues $\tilde{\omega}_{m}^{2}\left(\kappa^{2}\right)$ of $\hat{\mathcal{G}}_{\perp}$

For $m=1$, this yields $s_{1}\left(\kappa^{2}\right)=2$ which does not depend on $\kappa^{2}$. The explicit form of the eigenfunctions is thus

$$
\begin{aligned}
& \psi_{0}^{(\perp)}(y)=\psi_{0}^{(\perp)}\left(y ; \kappa^{2}\right)=N_{0}^{(\perp)} \operatorname{sech}^{s_{0}\left(\kappa^{2}\right)} y \\
& \psi_{1}^{(\perp)}(y)=N_{1}^{(\perp)} \operatorname{sech} y \tanh y \\
& \psi_{2}^{(\perp)}(y)=\psi_{2}^{(\perp)}\left(y ; \kappa^{2}\right)=N_{2}^{(\perp)} \operatorname{sech}^{\left(s_{2}\left(\kappa^{2}\right)-2\right)}(y)\left(1-\frac{2 s_{2}\left(\kappa^{2}\right)-1}{2 s_{2}\left(\kappa^{2}\right)-2} \operatorname{sech}^{2} y\right)
\end{aligned}
$$

where $N_{i}^{(\perp)}$ are appropriate normalisation constants. Like the zero eigenvalue, the zero mode (115) is independent of $\kappa^{2}$ and agrees with that given in the literature [20]. In the limit $\kappa^{2} \rightarrow 0$, we have $s_{0}(0)=2$, so $\psi_{0}^{(\perp)}\left(y ; \kappa^{2}=0\right)=\operatorname{sech}^{2} y$ which again is Mottola-Wipf's result. The $\kappa^{2} \rightarrow 0$ limit of $\psi_{2}^{(\perp)}$ is even more interesting, because there is no such third discrete eigenvalue in the Skyrme-less theory. In fact, we have $s_{2}(0)=2$ and therefore

$$
\lim _{|y| \rightarrow \infty} \lim _{\kappa^{2} \rightarrow 0} \psi_{2}^{(\perp)}\left(y ; \kappa^{2}\right)=1 \quad \Longrightarrow \quad \psi_{2}^{(\perp)}\left(\cdot ; \kappa^{2}=0\right) \notin L^{2}(\mathbb{R}) .
$$

But since $s_{2}\left(\kappa^{2}\right) \geq 2$ for $\kappa^{2} \geq 0$ and $\psi_{2}^{(\perp)}\left(y ; \kappa^{2}\right) \stackrel{|y| \rightarrow \infty, \kappa^{2}>0}{\longrightarrow} 0$ exponentially, $\psi_{2}^{(\perp)}$ is a square integrable eigenfunction (and $\omega_{2}^{2}$ really a discrete eigenvalue) for $\kappa^{2}>0$.

The continuous spectrum starts from $E>0$, i. e. $\omega^{2}(k)>\mu^{2}$. We denote the continuous eigenvalues by $\omega^{2}(k)=\mu^{2}\left(1+k^{2}\right), k \in \mathbb{R}$. The asymptotic behaviour of 
the scattering states belonging to $\omega^{2}(k)$ is discussed in Appendix B, (193 195), Now the parameter $s$ is not fixed but depends on $k$ (and $\kappa^{2}$ ):

$$
s=s_{\perp}\left(k ; \kappa^{2}\right)=\sqrt{\frac{25}{4}+4 \kappa^{2}\left(1+k^{2}\right)}-\frac{1}{2} \in \mathbb{R},
$$

and the coefficient of the incoming wave (195) is

$$
B\left(k ; \kappa^{2}\right)=\frac{\Gamma(-i k) \Gamma(1-i k)}{\Gamma\left(-i k-s\left(k ; \kappa^{2}\right)\right) \Gamma\left(-i k+s\left(k ; \kappa^{2}\right)+1\right)} .
$$

\subsection{Evaluation of the perpendicular fluctuation determinant}

With the above results we perform the discrete part of the product (96). From $m=2$, we simply get a harmonic oscillator factor

$$
\frac{1}{2 \sinh \left(\frac{\omega_{+}\left(\kappa^{2}\right) \beta_{T} \mu}{2}\right)}
$$

Next, we consider $m=0$. For sufficiently large $T$, there is only one negative factor in the product (95) which means that $\mathcal{G}_{\perp}^{(1)}$ has exactly one negative eigenvalue. Then we may interpret the negative mode as giving rise to an imaginary factor in $\mathcal{Z}_{1}$, according to the prescription

$$
\frac{1}{2 \sinh \left(\frac{\omega_{-}\left(\kappa^{2}\right) \beta_{T} \mu}{2}\right)} \longrightarrow \frac{1}{2} \frac{1}{2 i \sin \left(\frac{\left|\omega_{-}\left(\kappa^{2}\right)\right| \beta_{T} \mu}{2}\right)}
$$

where the additional factor $\frac{1}{2}$ arises from the distortion of the non-Gaussian contour over half of its range [35].

Finally, $m=0$ in $(95)$ is the zero mode contribution of $\tilde{\omega}_{1}^{2}=0$ which is discussed in Appendix A, yielding (169)

$$
\frac{1}{2 \sinh \frac{\tilde{\omega}_{1} \beta_{T}}{2}} \longrightarrow \mathcal{N}_{\perp}:=4 \sqrt{\lambda_{1}} \sqrt{\frac{\pi}{3 \beta_{T} \mu}} \sqrt{1+\frac{8}{5} \kappa^{2}}
$$

(since this zero mode results from the $U(2)$-rotation symmetry of the Lagrangian broken by the sphaleron, it does not lead to a space volume divergence like the translation zero mode factor (75); the parameter describing this symmetry takes values in $[0,2 \pi))$; cf. (155)).

The product over the continuum $\omega_{m}^{(\perp)}=\omega^{(\perp)}(k), k \in \mathbb{R}$ in (95) is rewritten as an integral over $\Phi\left(k^{2} ; \beta_{T}, \mu\right)$ defined in (64) weighted by the density $\rho_{\perp}\left(k ; \kappa^{2}\right)$ of the scattering states of eq. (97),

$$
\prod_{k \in \mathbb{R}} \frac{1}{2 \sinh \frac{\omega^{(\perp)}(k) \beta_{T}}{2}}=\exp \left(-\beta_{T} \int_{-\infty}^{\infty} d k \rho_{\perp}\left(k ; \kappa^{2}\right) \Phi\left(k^{2} ; \beta_{T}, \mu\right)\right)
$$


We are now able to write down the contribution of the perpendicular fluctuations to the transition rate. From (66) with $i=1$ and $(96,120,121,122,123)$ we have

$$
\begin{aligned}
\sqrt{\operatorname{det}\left(\frac{\hat{\mathcal{G}}_{\perp}^{(0)}}{\hat{\mathcal{G}}_{\perp}^{(1)}}\right)} & \frac{\mathcal{N}_{\perp}}{8 i \sin \left(\frac{\left|\omega_{-}\left(\kappa^{2}\right)\right| \beta_{T}}{2}\right) \sinh \left(\frac{\omega_{-}\left(\kappa^{2}\right) \beta_{T}}{2}\right)} \\
& \quad \times \exp \left(-\beta_{T} \int_{-\infty}^{\infty} d k\left(\rho_{\perp}\left(k ; \kappa^{2}\right)-\rho_{0}(k)\right) \Phi\left(k^{2} ; \beta_{T}, \mu^{2}\right)\right)
\end{aligned}
$$

The integral is performed using the analogue of formula (78),

$$
\int_{-\infty}^{\infty} d k\left(\rho_{\perp}\left(k ; \kappa^{2}\right)-\rho_{0}(k)\right) \Phi\left(k^{2} ; \beta_{T}, \mu\right)=\frac{1}{2 \pi} \int_{-\infty}^{\infty} d k \Phi\left(k^{2} ; \beta_{T}, \mu\right) \frac{d}{d k} \delta_{\perp}\left(k ; \kappa^{2}\right)
$$

where $\delta_{\perp}\left(k ; \kappa^{2}\right)$ is again the phase shift between incoming and outgoing plane waves in the scattering states of (97),

$$
\delta_{\perp}\left(k ; \kappa^{2}\right)=\arg \frac{\Gamma(-i k) \Gamma(1-i k)}{\Gamma\left(-i k-s_{\perp}\left(k ; \kappa^{2}\right)\right) \Gamma\left(-i k+s_{\perp}\left(k ; \kappa^{2}\right)+1\right)} .
$$

Using the decomposition $\Phi\left(k^{2} ; \beta_{T}, \mu\right)=\Phi_{1}\left(k^{2} ; \mu\right)+\frac{1}{\beta_{T}} \Phi_{2}\left(k^{2}, \beta_{T} \mu\right)$ (65), it is easy to show that the integral

$$
\int_{-\infty}^{\infty} d k \Phi_{1}\left(k^{2} ; \mu\right) \frac{d}{d k} \delta_{\perp}\left(k ; \kappa^{2}\right)
$$

is again a divergent zero point energy contribution which has to be renormalized. With $\Phi_{2}\left(k^{2}, \beta_{T} \mu\right)$, we define the function $\left(a=\beta_{T} \mu\right)$

$$
h_{\perp}\left(a, \kappa^{2}\right):=\frac{1}{2 \pi} \int_{-\infty}^{\infty} \ln \left(1-e^{-a \sqrt{1+k^{2}}}\right) \frac{d \delta_{\perp}\left(k ; \kappa^{2}\right)}{d k} d k
$$

which is discussed in Appendix Q. Its high temperature behaviour for $\kappa^{2}>0$ is given by (219),

$$
h_{\perp}\left(\beta_{T} \mu ; \kappa^{2}\right) \sim \frac{\pi m\left(\kappa^{2}\right)}{6} \frac{1}{\beta_{T} \mu}>0 \quad \text { for } \beta_{T} \mu \rightarrow 0 .
$$

This linear growth with temperature results from the large $k$ behaviour of the phase shift (211),

$$
-\delta_{\perp}\left(k ; \kappa^{2}\right) \lesssim m\left(\kappa^{2}\right) k+b\left(\kappa^{2}\right) \quad \text { for } k \rightarrow \infty
$$

In the Skyrme-less limit $\left(\kappa^{2}=0 \Rightarrow s_{\perp}\left(k ; \kappa^{2}=0\right)=2\right)$, this phase shift tends towards a constant, and the high temperature leading term of the continuum fluctuation contributions is again logarithmic (206),

$$
h_{\perp}\left(a ; \kappa^{2}=0\right) \sim-2 \ln \left(\beta_{T} \mu\right)>0 \quad \text { for } \quad \beta_{T} \mu \longrightarrow 0 .
$$


Finally, the perpendicular fluctuation contribution to the transition rate (59) is

$$
\sqrt{\operatorname{det}\left(\frac{\hat{\mathcal{G}}_{\perp}^{(0)}}{\hat{\mathcal{G}}_{\perp}^{(1)}}\right)}=\frac{\mathcal{N}_{\perp}}{8 i \sin \left(\frac{\left|\omega_{-}\left(\kappa^{2}\right)\right| \beta_{T} \mu}{2}\right) \sinh \left(\frac{\omega_{+}\left(\kappa^{2}\right) \beta_{T} \mu}{2}\right)} e^{-h_{\perp}\left(\beta_{T} \mu, \kappa^{2}\right)}
$$

\section{The thermal transition rate due to the sphale- ron: Skyrme-less limit and range of validity of the calculation}

So far we have evaluated explicitly the one-loop fluctuation contributions to the perturbative transition rate (59). The classical (zeroth order) part of this relation,

$$
\Gamma=-\frac{|\varrho|}{\pi} e^{-\beta_{T} \mathcal{E}\left[\vec{\varphi}_{1}\right]} \operatorname{Im} \sqrt{\operatorname{det}\left(\frac{\left(\hat{\mathcal{G}}_{\|}^{(0)}\right.}{\left(\hat{\mathcal{G}}_{\|}^{(1)}\right.}\right) \operatorname{det}\left(\frac{\hat{\mathcal{G}}_{\perp}^{(0)}}{\hat{\mathcal{G}}_{\perp}^{(1)}}\right)}
$$

is given by the exponential of the classical action of the sphaleron (51), i.e.

$$
S_{s p h}\left(\beta_{T}\right):=S_{e}\left[\vec{\varphi}_{1}\right]=\beta_{T} \lambda_{1} \int_{-\infty}^{\infty}\left[\frac{1}{2}\left(f^{\prime}(x)\right)^{2}+\mu^{2} V(\cos f(x))\right] d x=8 \beta_{T} \mu \lambda_{1} .
$$

Inserting now (82,132) and (134) into (133) (where the damping factor $|\varrho|$ for large $\lambda_{1}$ - which corresponds to the weak coupling limit of the original $O(3)-\sigma$ model may be approximated by the modulus of the negative eigenvalue, $\left.|\varrho|=\left|\omega_{-}\left(\kappa^{2}\right)\right|\right)$, the transition rate per unit volume given by the sphaleron is

$$
\frac{\Gamma}{L}=\frac{\left|\omega_{-}\left(\kappa^{2}\right)\right| \mu}{\pi} \cdot \frac{\frac{\lambda_{1}}{\sqrt{3} \beta_{T}} \sqrt{1+\frac{5}{8} \kappa^{2}}}{\sin \left(\frac{\left|\omega_{-}\left(\kappa^{2}\right)\right| \beta_{T} \mu}{2}\right) \sinh \left(\frac{\omega_{+}\left(\kappa^{2}\right) \beta_{T} \mu}{2}\right)} \cdot \exp \left\{-8 \beta_{T} \mu \lambda_{1}-h\left(\beta_{T} \mu ; \kappa^{2}\right)\right\}
$$

with the continuum fluctuation contributions described by the function

$$
h\left(\beta_{T} \mu ; \kappa^{2}\right):=h_{\|}\left(\beta_{T} \mu\right)+h_{\perp}\left(\beta_{T} \mu ; \kappa^{2}\right) .
$$

The prefactors of the exponential originate from the transition rate damping constant (first factor), the zero modes (numerator of the second factor) and the nonzero discrete modes of the fluctuation spectrum (denominator of the second factor).

The Skyrme-less limit of the model is given by $\kappa^{2} \rightarrow 0$. In this limit, there is no discrete eigenmode with positive eigenvalue $\omega_{+}\left(\kappa^{2}\right)$, the contribution $\frac{1}{\sinh \left(\frac{\omega_{+}\left(\kappa^{2}\right) \beta_{T} \mu}{2}\right)}$ being absorbed in the continuum fluctuation contribution,

$$
\frac{\exp \left\{-h\left(\beta_{T} \mu ; \kappa^{2}\right)\right\}}{2 \sinh \left(\frac{\omega_{+}\left(\kappa^{2}\right) \beta_{T} \mu}{2}\right)} \stackrel{\kappa^{2} \rightarrow 0}{\longrightarrow} \exp \left\{-h\left(\beta_{T} \mu ; \kappa^{2}=0\right)\right\}
$$


With $\left(\omega_{-}\left(\kappa^{2}=0\right)\right)^{2}=-3$, the Skyrme-less transition rate is given by

$$
\left.\frac{\Gamma}{L}\right|_{\kappa^{2}=0}=\frac{2 \lambda_{1} \mu}{\pi \beta_{T}} \frac{1}{\sin \left(\frac{\sqrt{3} \beta_{T} \mu}{2}\right)} \exp \left\{-8 \beta_{T} \mu \lambda_{1}-h\left(\beta_{T} \mu ; \kappa^{2}=0\right)\right\}
$$

which is exactly the result of Mottola and Wipf (eq. (5.10) in their paper), using our notation $\left(\lambda_{1} \leftrightarrow \frac{1}{g^{2}}, \omega^{2} \leftrightarrow \mu^{2}\right)$.

Next, we discuss the temperature range in which the sphaleron calculation is valid. We will see that this range is influenced by the Skyrme term. Since we performed a one-loop quantum approximation around the classical sphaleron solution using the idea that the imaginary part of the partition function describes transition rates, this range of validity is restricted by two technical requirements:

1. There has to be exactly one negative eigenvalue of the full Gaussian fluctuation operator. This leads to a lower bound.

2. The one loop fluctuation contributions must not be greater than the zeroth approximation given by the classical Boltzmann term. This leads to an upper bound.

The negative eigenvalue of the static fluctuation operator is (110), i.e.

$$
\omega_{-}^{2}\left(\kappa^{2}\right)=\frac{-\left(20 \kappa^{2}+\frac{11}{2}\right)+\frac{1}{2} \sqrt{25+112 \kappa^{2}+64\left(\kappa^{2}\right)^{2}}}{\left(1+4 \kappa^{2}\right)^{2}}<0
$$

and the corresponding eigenvalues of the full Gaussian fluctuation operator are given by

$$
\left(\frac{2 \pi n}{\beta_{T}}\right)^{2}+\left(\omega_{-}\left(\kappa^{2}\right)\right)^{2} \mu^{2} .
$$

$n=0$ always yields a negative eigenvalue, the eigenvalues for $n>0$ thus have to be nonnegative. Inserting $n=1$, one obtains from condition 1

$$
k_{B} T>\frac{\left|\omega_{-}\left(\kappa^{2}\right)\right| \mu}{2 \pi}=: k_{B} T^{(-)}\left(\mu, \kappa^{2}\right) .
$$

Without Skyrme term, this lower bound is $T^{(-)}\left(\mu, \kappa^{2}=0\right)=\frac{\sqrt{3}}{2 \pi} \mu$. With Skyrme term, it decreases with increasing Skyrme coupling (since $\left|\omega_{-}\left(\kappa^{2}\right)\right|$ decreases with increasing $\kappa)$. But for these lower temperatures, the exponential suppression due to the Boltzmann factor increases.

To analyse the second condition, one has to compare the classical action of the sphaleron, $S_{e}\left[\vec{\varphi}_{1}\right]=8 \beta_{T} \mu \lambda_{1}$ with the quantum effects of the linear fluctuations around it, given by the continuum fluctuation function $h$ in the exponent of (135) and the zero and discrete eigenmode factors in that formula. In fact, it is sufficient to compare $S_{e}\left[\vec{\varphi}_{1}\right]$ with $h\left(\beta_{T} \mu ; \kappa^{2}\right)$, because for high temperatures (small $\beta_{T}$ ), the discrete mode 
prefactors of (135) yield only logarithmic $\beta_{T} \mu$ contributions in the exponent, and $h$ itself has at least a logarithmic $\beta_{T} \mu \rightarrow 0$ behaviour. The upper temperature bound is thus given by the condition

$$
8 \beta_{T} \mu \lambda_{1} \gg h\left(\beta_{T} \mu ; \kappa^{2}\right)
$$

in which we ignore all numerical prefactors to obtain a rough estimate of the upper temperature bound $T^{(+)}\left(\mu, \lambda_{1} ; \kappa^{2}\right)$.

In the Skyrme-less case $\kappa^{2}=0$, the high temperature behaviour of $h$ is given by (81), (131), i.e. $h\left(\beta_{T} \mu, \kappa^{2}=0\right) \sim-3 \ln \left(\beta_{T} \mu\right), \beta_{T} \mu \rightarrow 0$. For small arguments, any

negative power function grows faster than the negative logarithm, $\lim _{a \rightarrow 0} \frac{(-\ln (a))}{a^{\gamma}}=0$ for all $\gamma>0$. This means that for any $\gamma>0$, there must exist a number $a^{*}(\gamma)$ such that for all $a<a^{*}(\gamma)$, we have $-\ln (a)<a^{-\gamma}$. Inserting this into (142) without numerical factors yields

$$
k_{B} T \ll \mu \lambda_{1}^{\frac{1}{\gamma+1}}
$$

The upper temperature limit therefore increases with $\lambda_{1}$ : High temperatures are allowed for large $\lambda_{1}$ which is the small coupling limit of the original $O(3)-\sigma$ model. This means we consider small $\beta_{T} \mu=a$ in the approximation of the logarithm $-\ln (a)<a^{-\gamma}$, therefore small $\gamma \neq 0$ are allowed in this approximation. Thus, the upper temperature bound in the "small coupling limit" $\lambda_{1} \rightarrow \infty$ is given by

$$
k_{B} T \ll \lambda_{1} \mu=: k_{B} T^{(+)}\left(\lambda_{1} \mu ; \kappa^{2}=0\right) .
$$

Next, including the Skyrme term changes the high temperature behaviour of $h$ drastically. In view of (129) we have

$$
h\left(\beta_{T} \mu ; \kappa^{2}>0\right) \sim \frac{\pi m\left(\kappa^{2}\right)}{6} \frac{1}{\beta_{T} \mu} \quad \text { for } \beta_{T} \mu \rightarrow 0 .
$$

Inserting this into (142) and again ignoring numerical prefactors immediateley yields

$$
k_{B} T \ll \mu \sqrt{\frac{\lambda_{1}}{m\left(\kappa^{2}\right)}}=: k_{B} T^{(+)}\left(\lambda_{1}, \mu ; \kappa^{2}>0\right)
$$

For fixed $\kappa^{2}>0$, this upper bound is considerably smaller than the Skyrme-less upper bound (143) in the "small coupling limit". One main effect of the Skyrme term in the sphaleron calculation of the thermal transition rate is thus to reduce the range of validity of the calculation. In the Skyrme-less limit, $T^{(+)}\left(\lambda_{1}, \mu ; \kappa^{2}>0\right)$ in eq. (145) increases as $m\left(\kappa^{2}\right)$ decreases for $\kappa^{2} \rightarrow 0$ which leads back to the Skyrme-less upper temperature bound condition (143).

\section{Discussion and conclusions}

After the detailed discussion of the sphaleron and the fluctuations around it in the Skyrmed reduced $O(3)-\sigma$ model we enquire about the physical implications of the results. 
First of all, from a very naive point of view, one might conclude that eq. (135) describes thermal transitions over the barrier separating the degenerate vacua which are exponentially suppressed at high temperatures. This would destroy the most important property of the sphaleron induced transition - namely that of not leading to exponential suppression - compared to that induced by the (vacuum) instanton which is always exponentially suppressed. But eq. (135) does not demand this interpretation, because in the temperature region where this exponential suppression becomes dominant, this formula is no longer valid for the reasons discussed in the last section.

At very high temperatures, $T>T^{(+)}$, the sphaleron calculation thus breaks down. In realistic models with spontaneous symmetry breaking (and particularly in the electroweak model), this is cured by the restoration of symmetry above $T_{\text {rest }} \approx T^{(+)}$ (which is indicated in the diagrams of Fig. 11 and Fig. 2). At these temperatures, the fluctuations become very large and the system becomes subject to disorder. It is clear that in this temperature range the transition rate becomes unsuppressed and there is no need to worry about the sphaleron which ceases to exist because it needs the broken symmetry. In $O(3)-\sigma$ models (with or without Skyrme term), where there is no Higgs-mechanism of spontaneous symmetry breaking, one has to add a symmetry breaking potential $\mu V\left(\phi^{3}\right)$ by hand. One can show that the explicitly broken symmetry of the $O(3)-\sigma$ model is not restored at high temperatures, so that for very high temperatures $T>T^{(+)}$, both the Skyrme-less and the Skyrmed reduced $O(3)-\sigma$ model cease to be physically meaningful theories. The only difference in the very high temperature behaviour is therefore that $T^{(+)}$decreases with increasing Skyrme coupling $\kappa^{2}$, but this is what one expects on physical grounds, because at high temperatures, the Skyrme term (having the highest number of derivatives in the Lagrangian) dominates the theory with large fluctuation effects which would lead to symmetry restoration also at lower temperatures in a realistic theory.

Finally, we consider the situation in the lower temperature region around $T^{(-)}$. As already remarked in the introduction, the Skyrme-less limit of the model (i.e. the original reduced $O(3)-\sigma$ model) is a type II theory with dominant transition processes as indicated in Fig. 2. This changes completely for $\kappa^{2}>0$ since the Skyrme term enables the existence of localised instantons with Euclidean energy (3) [15]

$$
2 S_{0}=8 \pi \lambda_{1} w(\kappa), \quad w(\kappa)=1+\mathcal{O}(\kappa)
$$

(the function $w(\kappa)$ has to be evaluated numerically since the vacuum instanton is not known explicitly [21]) and corresponding periodic instantons with $\frac{\partial \mathcal{E}_{\text {inst }}}{\partial \beta_{T}}<0$.In the limit $\kappa^{2} \rightarrow 0$, there is thus a discontinuous change in the type of instantons involved (whereas the action of the vacuum instanton is continuous for $\kappa^{2} \rightarrow 0$, the action of the constrained instanton in the Skyrme-less theory can be estimated to be $2 S_{0}=$ $8 \pi \lambda_{1}$ [14). In the case of the sphaleron of the Skyrmed model, there is a smooth crossover to the Skyrme-less situation. The sphaleron is only quantitatively changed by the Skyrme term, whereas the instanton changes qualitatively. In particular, the 
Euclidean action $S_{s p h}^{(-)}$of the sphaleron at the lower temperature limit $T^{(-)}$,

$$
S_{s p h}^{(-)}=\frac{16 \pi \lambda_{1}}{\left|\omega_{-}\left(\kappa^{2}\right)\right|}
$$

depends smoothly on the Skyrme coupling $\kappa^{2}$. This means that the type II condition

$$
2 S_{0}<S_{s p h}^{(-)} \Leftrightarrow T_{0}>T^{(-)}
$$

which is true for $\kappa^{2}=0$ remains true for $\kappa^{2}>0$ due to the smooth $\kappa^{2}$-dependence of both actions, whereas the type of instantons changes discontinuously from those in type II theories to those in type I theories. The Skyrmed reduced $O(3)-\sigma$ model is therefore neither of type I nor of type II at least for a weak Skyrme coupling [15]. Whether it may become a type I theory with increasing Skyrme coupling depends on the function $w(\kappa)$ which would have to satisfy the condition $w(\kappa)\left|\omega_{-}\left(\kappa^{2}\right)\right|>2$ for some $\kappa^{2}$. But this is very unlikely to happen, because as shown in Fig. 3 , $\left|\omega_{-}\left(\kappa^{2}\right)\right|$ decreases rapidly to 0 as $\kappa^{2}$ increases.

\section{Acknowledgements}

This work was supported in part by a Forbairt(Ireland) grant, project No. IC/97/019.

The authors are indebted to A. Wipf (Jena) for detailed discussions and to V.A. Rubakov (Moscow) also for a critical reading of the manuscript with valuable comments.

\section{Appendix}

\section{A Zero modes of the $O(3)$ model}

The spectra of the operators $\mathcal{H}_{\|}, \mathcal{H}_{\perp}$ each contain one zero eigenvalue which appears in the evaluation of the fluctuation integrals (56,57) and yields divergences in (70, 95). In this Appendix, we discuss the origin of these "zero modes" 229.

For this reason we analyse the symmetries of the model (1) which are broken by the classical solution $\vec{\varphi}_{1}(x)=(\sin f(x), 0, \cos f(x))$. We consider the following symmetries of the model (1):

1. space translations:

$$
\begin{aligned}
\mathbf{T}[\vec{\phi}, a](\tau, x) & =\vec{\phi}(\tau, x+a) \\
& =\vec{\phi}(\tau, x)+\frac{\partial \vec{\phi}}{\partial x}(\tau, x) \cdot a+\mathcal{O}\left(a^{2}\right) \text { for } a \rightarrow 0
\end{aligned}
$$

(There is also a time translation symmetry, but this is not broken by a static solution.) 
2. internal $S O(2)$-rotations:

$$
\begin{aligned}
\mathbf{R}[\vec{\phi}, \alpha](\tau, x) & =\left(\begin{array}{ccc}
\cos \alpha & -\sin \alpha & 0 \\
\sin \alpha & \cos \alpha & 0 \\
0 & 0 & 1
\end{array}\right) \vec{\phi}(\tau, x) \\
& =\vec{\phi}(\tau, x)+\left(\begin{array}{ccc}
0 & -1 & 0 \\
1 & 0 & 0 \\
0 & 0 & 1
\end{array}\right) \vec{\phi}(\tau, x) \cdot \alpha+\mathcal{O}\left(\alpha^{2}\right) \quad \text { for } \quad \alpha \rightarrow 0
\end{aligned}
$$

The classical solution $\vec{\varphi}_{1}(x)=(\sin f(x), 0, \cos f(x))$ breaks this invariance because

$$
\mathbf{T}\left[\vec{\varphi}_{1}, a\right](\tau, x) \equiv \vec{\varphi}_{1}(x+a) \neq \vec{\varphi}_{1}(x), \quad \mathbf{R}\left[\vec{\varphi}_{1}, \alpha\right](\tau, x) \neq \vec{\varphi}_{1}(x)
$$

The moduli space [36] of the parameters $a, \alpha$ describing finite energy solutions is therefore topologically $\mathcal{M}=\mathbb{R}^{1} \times \mathrm{S}^{1}$ and one can show that $\frac{\partial \mathbf{T}}{\partial a}\left[\vec{\varphi}_{1}, 0\right](x)$ and $\frac{\partial \mathbf{R}}{\partial \alpha}\left[\vec{\varphi}_{1}, 0\right](x)$ are zero modes of the static Gaussian fluctuation operator $\left[\frac{\delta^{2} \mathcal{E}[\vec{\phi}]}{\delta \phi^{i}(x) \delta \phi^{j}\left(x^{\prime}\right)}\right]_{\vec{\varphi}}$ by expanding

$$
\mathcal{E}\left[\vec{\varphi}_{1}+\frac{\partial \mathbf{T}}{\partial a}\left[\vec{\varphi}_{1}, 0\right]\right] \quad \text { and } \mathcal{E}\left[\vec{\varphi}_{1}+\frac{\partial \mathbf{R}}{\partial a}\left[\vec{\varphi}_{1}, 0\right]\right]
$$

around $\vec{\varphi}_{1}$. The problem here is that one has to be very careful with the constraint $\vec{\phi}^{2}=1$, and it is better to consider the broken symmetries in the parametrization $\vec{\varphi}(\vec{f})$. Therefore we first express the symmetries (150), (151) in terms of the parameter fields. This is done by expanding the ansatz (17) in small quantities $\delta \vec{f}$ around $\vec{f}=\left(f^{(\|)}, f^{(\perp)}\right)$ and then comparing the result with the infinitesimal form of the symmetries given by (150), (151), also written in terms of the parameter fields. The result is

1. for space translations:

$$
\mathbf{T}_{f}[\vec{f}, a](\tau, x)=\vec{f}(\tau, x)+\frac{\partial \vec{f}}{\partial x}(\tau, x) \cdot a+\mathcal{O}\left(a^{2}\right) \quad \text { for } \quad a \rightarrow 0
$$

2. for internal $S O(2)$-rotations:

$$
\begin{array}{r}
\mathbf{R}_{f}[\vec{f}, \alpha](\tau, x)=\vec{f}(\tau, x)+\left(\begin{array}{c}
-f^{(\perp)}(\tau, x) \cos f^{(\|)}(\tau, x) \\
\left(1+\left(f^{(\perp)}(\tau, x)\right)^{2}\right) \sin f^{(\|)}(\tau, x)
\end{array}\right) \cdot \alpha+\mathcal{O}\left(\alpha^{2}\right) \\
\text { for } \quad \alpha \rightarrow 0(155)
\end{array}
$$


In terms of the parametrization $\vec{f}$, the classical solution $\vec{\varphi}$ is given by $\vec{f}(x)=\vec{f}_{0}(x)=$ $(f(x), 0)$. This yields the following two zero modes of the Gaussian fluctuation operator in $\vec{f}$-parametrization,

$$
\begin{aligned}
& \frac{\partial \mathbf{T}_{f}}{\partial a}\left[\overrightarrow{f_{0}}, 0\right]=\left(\begin{array}{c}
f^{\prime} \\
0
\end{array}\right), \quad f^{\prime}(x)=2 \mu \operatorname{sech}(\mu x) \\
& \frac{\partial \mathbf{R}_{f}}{\partial \alpha}\left[\overrightarrow{f_{0}}, 0\right]=\left(\begin{array}{c}
0 \\
\sin f
\end{array}\right), \quad \sin f(x)=-2 \operatorname{sech}(\mu x) \tanh (\mu x)
\end{aligned}
$$

Obviously, they are proportional to the zero eigenvalue eigenfunctions of the fluctuation equations (69,86), normalised with respect to the appropriate scalar products $(71,87)$,

$$
\begin{aligned}
& \psi_{0}^{(\|)}(x)=\sqrt{\frac{\mu}{2}} \operatorname{sech}(\mu x), \quad\left\langle\psi_{0}^{(\|)}, \psi_{0}^{(\|)}\right\rangle_{\|}=1 \\
& \psi_{1}^{(\perp)}(x)=\sqrt{\frac{15 \mu}{10+16 \kappa^{2}}} \operatorname{sech}(\mu x) \tanh (\mu x), \quad\left\langle\psi_{1}^{(\perp)}, \psi_{1}^{(\perp)}\right\rangle_{\perp}=1
\end{aligned}
$$

Next, we need a convenient way to take these zero modes into account in the Fourier-Matsubara evaluation of the fluctuation integrals (70,95). Usually, on uses the method of collective coordinates which means that one treats the parameters which describe the breakdown of a global symmetry of the theory in a static solution ( $a$ and $\alpha$ in the case of the $O(3)$ model) as additional dynamical degrees of freedom, i.e. one considers

$$
\vec{\phi}(\tau, x)=\mathbf{R}[\mathbf{T}[\vec{\varphi}, a(\tau)], \alpha(\tau)](\tau, x)
$$

and thus adds two redundant degrees of freedom $a, \alpha$. One can show that the resulting theory is a gauge theory, i. e. the Euclidean action of the theory in terms of the new "fields" $S_{e}[\vec{v}, a, \alpha]$ ( $\vec{v}$ denoting again the parameter fields of the fluctuations) is invariant under some (time-)local transformations of $\vec{v}, a, \alpha$. Therefore, the usual techniques for path-integral quantization of gauge theories (Faddeev-Popov [37 or BRST 38]) can be applied to evaluate the partition function $\mathcal{Z}_{1}$ around $\vec{\varphi}_{1}$. A convenient gauge-fixing condition is to exclude fluctuations parallel to the zero modes. This procedure is well-known and discussed in detail in the literature, even in the particular context of $O(3)$ models, using classical Faddeev-Popov [39] or more modern BRST and BV methods [40, 41]. Therefore, we do not discuss the details.

Here, we use a simpler semiclassical method [20] to compute the zero mode contributions to the partition function, i.e. to treat them in fluctuation integrals (70,95). In the harmonic oscillator decomposition of the Gaussian fluctuation operators $\mathcal{G}_{\|}^{(1)}$, $\mathcal{G}_{\perp}^{(1)}$, each zero mode contributes one harmonic oscillator mode with zero frequency, i. e. a free mode. The classical contribution of a free mode to the partition function is

$$
\int \frac{d q d p}{2 \pi} e^{-\beta_{T} \frac{p^{2}}{2}}=\frac{1}{\sqrt{2 \pi \beta_{T}}} \int d q
$$


The "coordinate" $q$ of this free mode has to be identified with the zero mode coefficients $c_{0}^{(\|)}, c_{1}^{(\perp)}$ in the harmonic oscillator decompositions of the parallel and perpendicular fluctuations, (72) and (88), respectively. Therefore, we consider the fluctuations in direction of the zero modes. Let $\mathbf{p r}_{0}$ be the projector from the space of fluctuations $\vec{v}(\tau, x)$ on the subspace of zero mode fluctuations. Since the zero modes are those which result from fluctuations in the collective coordinates, expanding the parameter field version of $(160)$

$$
\vec{f}(\tau, x)=\mathbf{R}_{f}\left[\mathbf{T}_{f}\left[\overrightarrow{f_{0}}, a(\tau)\right], \alpha(\tau)\right](\tau, x)
$$

around the classical static solution $\overrightarrow{f_{0}}=\mathbf{R}_{f}\left[\mathbf{T}_{f}\left[\overrightarrow{f_{0}}, 0\right], 0\right]$ (small $a, \alpha$ ) yields

$$
\begin{aligned}
\vec{f}(\tau, x)= & \mathbf{R}_{f}\left[\mathbf{T}_{f}\left[\vec{f}_{0}, 0\right], 0\right](\tau, x) \\
& +\mathbf{R}_{f}\left[\frac{d \mathbf{T}_{f}}{d a}\left[\overrightarrow{f_{0}}, 0\right], 0\right](\tau, x) \cdot a(\tau)+\frac{d \mathbf{R}_{f}}{d \alpha}\left[\mathbf{T}_{f}\left[\vec{f}_{0}, 0\right], 0\right](\tau, x) \cdot \alpha(\tau) \\
= & \overrightarrow{f_{0}}(x)+\frac{d \mathbf{T}_{f}}{d a}\left[\vec{f}_{0}, 0\right](\tau, x) \cdot a(\tau)+\frac{d \mathbf{R}_{f}}{d \alpha}\left[\vec{f}_{0}, 0\right](\tau, x) \cdot \alpha(\tau) \\
= & \overrightarrow{f_{0}}(x)+\sqrt{\lambda_{1}} \mathbf{p r}_{0}\{\vec{v}(\tau, x)\}
\end{aligned}
$$

with

$$
\mathbf{p r}_{0}\{\vec{v}(\tau, x)\}=\frac{d \mathbf{T}_{f}}{d a}\left[\vec{f}_{0}, 0\right](\tau, x) \cdot a(\tau)+\frac{d \mathbf{R}_{f}}{d \alpha}\left[\vec{f}_{0}, 0\right](\tau, x) \cdot \alpha(\tau) .
$$

(The factor $\lambda_{1}$ appears because an overall factor $\lambda_{1}$ is extracted from the fluctuation expansion (48 50)).

But from the decompositions (72) and (88), the parallel and perpendicular components of the projection operator $\mathbf{p r}_{0}$ are given by $\mathbf{p r}_{0}^{(\|)}=\left\langle\cdot, \psi_{0}^{(\|)}\right\rangle_{\|} \psi_{0}^{(\|)}$and $\mathbf{p r}_{0}^{(\perp)}=\left\langle\cdot, \psi_{1}^{(\perp)}\right\rangle_{\perp} \psi_{1}^{(\perp)}$, so that with $(156,157)$ :

$$
\sqrt{\lambda_{1}} \mathbf{p r}_{0}\{\vec{v}(\tau, x)\}=\sqrt{\lambda_{1}}\left(\begin{array}{c}
c_{0}^{(\|)}(\tau) \psi_{0}^{(\|)}(x) \\
c_{1}^{(\perp)}(\tau) \psi_{1}^{(\perp)}(x)
\end{array}\right)=\left(\begin{array}{c}
a(\tau) f^{\prime}(x) \\
\alpha(\tau) \sin f(x)
\end{array}\right) .
$$

Thus we have

$$
\begin{aligned}
& \int d q \longrightarrow \sqrt{\lambda_{1}} \int d c_{0}^{(\|)}=\sqrt{\lambda_{1}} \Delta c_{0}^{(\|)}=\sqrt{\lambda_{1}} \sqrt{\left\langle f^{\prime}, f^{\prime}\right\rangle_{\|}} \Delta a \\
& \int d q \longrightarrow \sqrt{\lambda_{1}} \int d c_{1}^{(\perp)}=\sqrt{\lambda_{1}} \Delta c_{1}^{(\perp)}=\sqrt{\lambda_{1}} \sqrt{\langle\sin f, \sin f\rangle_{\perp}} \Delta \alpha
\end{aligned}
$$

with

$$
\left\langle f^{\prime}, f^{\prime}\right\rangle_{\|}=4 \mu^{2}, \quad\langle\sin f, \sin f\rangle_{\perp}=\frac{10+16 \kappa^{2}}{15} \frac{4}{\mu}
$$

$\Delta a, \Delta \alpha$ are the values the parameters describing the broken symmetry can take: For rotations, it is clear that $\Delta \alpha=2 \pi$. To avoid a volume divergence due to the translation parameter $a \in \mathbb{R}$, we restrict the model to a finite space volume such that $\Delta a=L$. Therefore the zero mode factors are

$$
L \mathcal{N}_{\|}=2 L \sqrt{\lambda_{1}} \sqrt{\frac{\mu}{\pi \beta_{T}}}, \quad \mathcal{N}_{\perp}=4 \sqrt{\lambda_{1}} \sqrt{\frac{\pi}{3 \beta_{T} \mu}} \sqrt{1+\frac{8}{5} \kappa^{2}} .
$$




\section{B The Schrödinger equation with $\operatorname{sech}^{2}$-potential}

All attempts to evaluate the fluctuation factors $(56,57)$ lead to the eigenvalue differential equation

$$
\psi^{\prime \prime}+\left(E+U \operatorname{sech}^{2} y\right) \psi=0
$$

with $\psi=\psi(y)$ which we discuss in this section [42]. There are three different cases to be distinguished:

1. $U>0$ : then it is possible to have

(a) $E<0$, i. e. bound states (discrete spectrum)

(b) $E>0$, i. e. scattering states (continuous spectrum)

2. $U<0$, then $E>0$ and only scattering states (continuous spectrum) exist.

We first set $\xi=\tanh y$ to get (now $\psi=\psi(\xi))$ :

$$
\frac{d}{d \xi}\left[\left(1-\xi^{2}\right) \frac{d \psi}{d \xi}\right]+\left(U+\frac{E}{1-\xi^{2}}\right) \psi=0
$$

Assuming $U>0$, we first compute the discrete spectrum for which $E<0$ is valid. We may thus define

$$
\epsilon:=\sqrt{-E}, \quad \epsilon>0
$$

and

$$
U=s(s+1), \quad s=-\frac{1}{2}+\sqrt{\frac{1}{4}+U}>0
$$

to obtain from (171) the differential equation of generalized Legendre functions,

$$
\frac{d}{d \xi}\left[\left(1-\xi^{2}\right) \frac{d \psi}{d \xi}\right]+\left[s(s+1)-\frac{\epsilon^{2}}{1-\xi^{2}}\right] \psi=0
$$

Next we set $\psi(\xi)=\left(1-\xi^{2}\right)^{\frac{\epsilon}{2}} w(\xi)$ which yields

$$
\left(1-\xi^{2}\right) \frac{d^{2} w}{d \xi^{2}}-2(\epsilon+1) \frac{d w}{d \xi}+\left[s(s+1)-\epsilon-\epsilon^{2}\right] w=0
$$

Finally, changing the argument to $\chi=\frac{1}{2}(1-\xi)$, (175) transforms to

$$
\chi(\chi-1) \frac{d^{2} w}{d \chi^{2}}+[(\epsilon+1)-(2 \epsilon+2) \chi] \frac{d w}{d \chi}-(\epsilon-s)(\epsilon+s+1) w=0
$$

which is a hypergeometric differential equation.

The general form of the hypergeometric differential equation is

$$
z(z-1) \frac{d^{2} \phi}{d z^{2}}+\left[(c-(a+b+1) z] \frac{d \phi}{d z}-a b \phi=0\right.
$$


Solutions are given by the hypergeometric functions

$$
F(a, b, c ; z)=\sum_{k=0}^{\infty} \frac{(a)_{k}(b)_{k}}{(c)_{k}} \frac{z^{k}}{k !}
$$

with

$$
(a)_{k}=\frac{\Gamma(a+k)}{\Gamma(a)}=a(a+1) \cdot \ldots \cdot(a+k-1), \quad(a)_{0}=1 .
$$

The series (178) converges for $|z|<1, z \in \mathbb{C}$. It also converges for $z=1$ if $c-a-b>0$.

Comparing (176) with the general form (177), we identify $a=\epsilon-s, b=\epsilon+s+1$ and $c=\epsilon+1$, so the solution to eq. (176) is given by

$$
w(\chi)=F(\epsilon-s, \epsilon+s+1, \epsilon+1 ; \chi) .
$$

Inserting all the substitutions, the solution to the original equation (170) may be written as

$$
\psi(y)=\left(1-\tanh ^{2} y\right)^{\frac{\epsilon}{2}} F\left(\epsilon-s, \epsilon+s+1, \epsilon+1 ; \frac{1}{2}(1-\tanh y)\right) .
$$

We want to compute bound states (discrete eigenvalues for $E<0$ ). Hence the solutions (180) have to be square integrable which means that at least (as all functions are continuous) $\psi(y) \stackrel{|y| \rightarrow \infty}{\longrightarrow} 0$. In terms of the $\chi$-variable, which is related to the original variable $y$ by $\chi=\frac{1}{2}(1-\tanh y)$, this means that $w(\chi)$ has to be bounded as $\chi \stackrel{y \rightarrow \infty}{\longrightarrow} 0$ and $\stackrel{y \rightarrow-\infty}{\longrightarrow} 1$. We always have $F(a, b, c ; 0)=1$, so the limit $y \rightarrow \infty$ is no problem. But since $c-a-b=-\epsilon<0, F(a, b, c ; 1)$ is not bounded for general values of $a, b, c$. We thus have to force $F(\epsilon-s, \epsilon+s+1, \epsilon+1 ; 1)<\infty$ which is achieved by making the infinite sum (178) finite: $(m)_{k}=0$ for $-m \in \mathbb{N}_{0}$ and $k>m$. This yields the quantization condition $\epsilon-s=-m \in N_{0}$ : $m$ labels the eigenvalues of (174), $\epsilon_{m}=s-m$. There are no eigenvalues for any $m \in N_{0}$ in view of the condition (172) $\epsilon>0$ which means $m<s$. The discrete spectrum of eq. (170) (case 1a) is therefore given by

$$
\left\{E_{m}=-\left(\sqrt{\frac{1}{4}+U}-\frac{1}{2}-m\right)^{2} \mid m \in N_{0} \quad \text { and } \quad T_{U}(m)>0\right\}
$$

with

$$
T_{U}(m):=\sqrt{\frac{1}{4}+U}-\frac{1}{2}-m,
$$

and the corresponding eigenfunctions are (with $1-\tanh ^{2} y=\operatorname{sech}^{2} y$ )

$$
\psi_{m}(y)=\operatorname{sech}^{(s-m)}(y) F\left(-m, 2 s-m+1, s-m+1 ; \frac{1}{2}(1-\tanh y)\right) .
$$


Next, we assume $U<0$. Then, there is only a positive, continuous spectrum (case 2) with $E>0$, and we substitute

$$
\begin{gathered}
k:= \pm \sqrt{E} \Longrightarrow \quad( \pm i k)^{2}=-E \\
U=s(s+1), \quad s=-\frac{1}{2}+\sqrt{\frac{1}{4}+U} \in \mathbb{C} .
\end{gathered}
$$

Performing the same steps as above, the scattering states of eq. (170) for $U<0$ are given by

$$
\psi_{ \pm, k}(y)=\left(1-\tanh ^{2} y\right)^{ \pm \frac{i k}{2}} F\left( \pm i k-s, \pm i k+s+1, \pm i k+1 ; \frac{1}{2}(1-\tanh y)\right)
$$

and the continuous spectrum is

$$
\left\{E=k^{2} \mid k \in \mathbb{R}\right\} \text {. }
$$

It is obvious that these calculations are also true for $U>0, E>0$ (then, $s$ is real), so that (187) also describes the scattering states of the $U>0$ potential which has bound and scattering eigenstates (case 1b).

Next, we analyse the asymptotic behaviour of the scattering states (187). It will be sufficient to consider the scattering state $\psi_{-, k}(y)$. The prefactor in (187) behaves like

$$
\left(1-\tanh ^{2} y\right)^{-\frac{i k}{2}} \sim 4^{\frac{i k}{2}} e^{ \pm i k} \quad \text { for } \quad x \longrightarrow \pm \infty
$$

(we ignore the common factor $4^{\frac{i k}{2}}$ in the following.) To deal with the hypergeometric function, we use $(178)$, i. e. $F(a, b, c ; z)=1+\mathcal{O}(z)$ for $z \rightarrow 0$. This works well for $y \rightarrow+\infty$, because then $\frac{1}{2}(1-\tanh y) \rightarrow 0$. For $y \rightarrow-\infty$, we use the following identity:

$$
\begin{aligned}
F(a, b, c ; z)= & \frac{\Gamma(c) \Gamma(c-a-b)}{\Gamma(c-a) \Gamma(c-b)} F(a, b, a+b+1-c ; 1-z) \\
& +\frac{\Gamma(c) \Gamma(a+b-c)}{\Gamma(a) \Gamma(b)}(1-z)^{c-a-b} F(c-a, c-b, c+1-a-b ; 1-z)
\end{aligned}
$$

which yields, applied to (187)

$$
\begin{aligned}
& F\left(-i k-s,-i k+s+1,-i k+1 ; \frac{1}{2}(1-\tanh y)\right) \\
& =\frac{\Gamma(-i k+1) \Gamma(i k)}{\Gamma(-s) \Gamma(1+s)} F\left(-i k-s,-i k+s+1,-i k+1 ; \frac{1}{2}(1+\tanh y)\right) \\
& +\frac{\Gamma(-i k+1) \Gamma(-i k)}{\Gamma(-i k-s) \Gamma(-i k+s+1)}\left(\frac{1}{2}(1+\tanh y)\right)^{i k} \\
& \quad \times F\left(s+1,-s, i k+1 ; \frac{1}{2}(1+\tanh y)\right) .
\end{aligned}
$$


Inserting

$$
\frac{1}{2}(1 \pm \tanh y) \sim e^{ \pm 2 y} \quad \text { for } \quad y \longrightarrow \mp \infty
$$

and putting everything together, we get the following asymptotic behaviour of the scattering states:

$$
\begin{array}{ll}
\psi_{-, k}(y) & \stackrel{y \rightarrow-\infty}{\sim} A(k) e^{-i k y}+B(k) e^{i k y} \\
\psi_{-, k}(y) & \stackrel{y \rightarrow+\infty}{\sim} e^{i k y} .
\end{array}
$$

with

$$
A(k)=\frac{\Gamma(i k) \Gamma(1-i k)}{\Gamma(-s) \Gamma(1+s)} \quad B(k)=\frac{\Gamma(-i k) \Gamma(1-i k)}{\Gamma(-i k-s) \Gamma(-i k+s+1)} .
$$

Since $k^{2}>0$, the poles of $B(k)$ are excluded. $A(k)$ vanishes for $s \in \mathbb{N}$. This is the well-known case of the reflection free Rosen-Morse potential.

Eqs. (193, 194) show that $\psi_{-, k}$ describes incoming particles from the left; there are no reflected particles to the right (one can check that the second independent solution $\psi_{+, k}$ describes incoming particles from the right). The incoming flux is normalised to unity. Thus, all important information about the scattering solutions resides in the phase shift $\delta$ defined by the transmission coefficient $B(k) \equiv e^{i \delta}$ :

$$
\delta=\delta_{s}(k)=\arg \frac{\Gamma(-i k) \Gamma(1-i k)}{\Gamma(-i k-s) \Gamma(-i k+s+1)} .
$$

For $s \in \mathbb{N}$, there is a simple formula for $\delta_{s}(k)$ 31]:

$$
\delta_{s}(k)=-2 \sum_{n=1}^{s} \arctan \left(\frac{k}{n}\right) \text {. }
$$

\section{C (High) temperature behaviour of the functions $h_{\|}$and $h_{\perp}$}

In this Appendix, we analyse the small $a=\beta_{T} \mu$ behaviour of the functions $h_{\|}$(80), $h_{\perp}$ (128) given by the integrals

$$
\frac{1}{2 \pi} \int_{-\infty}^{\infty} \ln \left(1-e^{-a \sqrt{1+k^{2}}}\right) \frac{d \delta_{s_{\|, \perp}}(k)}{d k} d k
$$

with 196$)$

$$
\delta_{s}(k):=\arg \frac{\Gamma(-i k) \Gamma(1-i k)}{\Gamma(-i k-s) \Gamma(-i k+s+1)} .
$$

$\delta_{s}$ is known for arbitrary values of $s$, but we will only use it for integer $s \in \mathbb{N}$ (cf. formula (197)). 
For the parallel fluctuation continuum, we have $s_{\|} \equiv 1$ and $\frac{d \delta_{1}(k)}{d k}=-\frac{2}{1+k^{2}}$ so that

$$
h_{\|}(a)=-\frac{1}{\pi} \int_{-\infty}^{\infty} \frac{\ln \left(1-e^{-a \sqrt{1+k^{2}}}\right)}{1+k^{2}} d k .
$$

This integral is convergent for $a>0$. For high temperatures we have $a=\beta_{T} \mu \rightarrow 0$. Using the approximation

$$
\ln \left(1-e^{-a \sqrt{1+k^{2}}}\right) \approx \ln \left(a \sqrt{1+k^{2}}\right)=\frac{1}{2} \ln \left(a^{2}+(a k)^{2}\right),
$$

the substitution $\zeta=a k$ yields

$$
h_{\|}(a) \approx-\frac{a}{2 \pi} \int_{-\infty}^{\infty} \frac{\ln \left(a^{2}+\zeta^{2}\right)}{a^{2}+\zeta^{2}} d \zeta=-\ln (2 a)
$$

which means

$$
h_{\|}\left(\beta_{T} \mu\right) \sim-\ln \left(\beta_{T} \mu\right)>0 \quad \text { for } \quad \beta_{T} \mu \longrightarrow 0 .
$$

In the case of perpendicular fluctuations, the behaviour of the phase shift is much more complicated. We have $\delta_{s_{\perp}}(k)=\delta_{\perp}\left(k ; \kappa^{2}\right)$ because (cf. eq. (118))

$$
s_{\perp}=s_{\perp}\left(k ; \kappa^{2}\right)=\sqrt{\frac{25}{4}+4 \kappa^{2}\left(1+k^{2}\right)}-\frac{1}{2} .
$$

The Skyrme-less limit $\left(\kappa^{2}=0\right)$ reduces this to $s_{\perp}\left(k ; \kappa^{2}=0\right)=2$ and $\frac{d \delta_{2}(k)}{d k}=$ $-\frac{2}{1+k^{2}}-\frac{4}{4+k^{2}}$, and one can use the same approximations as in the parallel case which yield

$$
\begin{aligned}
h_{\perp}\left(a ; \kappa^{2}=0\right) & =-\frac{1}{\pi} \int_{-\infty}^{\infty} \ln \left(1-e^{-a \sqrt{1+k^{2}}}\right)\left[\frac{1}{1+k^{2}}+\frac{2}{4+k^{2}}\right] d k \\
& \stackrel{\zeta=a k}{\approx}-\frac{a}{2 \pi} \int_{-\infty}^{\infty} \ln \left(a^{2}+\zeta^{2}\right)\left[\frac{1}{a^{2}+\zeta^{2}}+\frac{2}{4 a^{2}+\zeta^{2}}\right] d \zeta \\
& =-(\ln (2 a)+\ln (3 a))
\end{aligned}
$$

and thus

$$
h_{\perp}\left(a ; \kappa^{2}=0\right) \sim-2 \ln \left(\beta_{T} \mu\right)>0 \quad \text { for } \quad \beta_{T} \mu \longrightarrow 0 .
$$

Including the Skyrme term $\left(\kappa^{2}>0\right)$ changes this behaviour. First, in order to restrict the following discussion to positive $k$, we perform an integration by parts in (198) and use the symmetry of the integrand to obtain

$$
h_{\perp}\left(a ; \kappa^{2}\right)=\frac{1}{\pi} \int_{0}^{\infty}\left[-\delta_{\perp}\left(k ; \kappa^{2}\right)\right] \frac{d}{d k}\left[\ln \left(1-e^{-a \sqrt{1+k^{2}}}\right)\right] d k .
$$

For fixed $k, s_{\perp}\left(k ; \kappa^{2}\right)$ and thus $-\delta_{\perp}\left(k ; \kappa^{2}\right)$ increase with $\kappa^{2}$, so $h_{\perp}\left(a ; \kappa^{2}\right)$ increases with increasing Skyrme coupling $\kappa^{2}$ for fixed $a$. Now the phase shift (196), i.e.

$$
\delta_{\perp}\left(k ; \kappa^{2}\right)=\arg \frac{\Gamma(-i k) \Gamma(1-i k)}{\Gamma\left(-i k+\frac{1}{2}-\sqrt{\frac{25}{4}+4 \kappa^{2}\left(1+k^{2}\right)}\right) \Gamma\left(-i k+\frac{1}{2}+\sqrt{\frac{25}{4}+4 \kappa^{2}\left(1+k^{2}\right)}\right)}
$$


can be evaluated numerically. The main result is that for fixed $\kappa^{2}$, one can always find positive real numbers $\alpha=\alpha\left(\kappa^{2}\right)>1, c=c\left(\kappa^{2}\right)>0$ such that

- for $k \in(0, \alpha),\left|\delta_{\perp}\left(k ; \kappa^{2}\right)\right|$ is bounded by $c<\infty$

- for $k \geq \alpha,-\delta_{\perp}\left(k ; \kappa^{2}\right)$ increases linearly.

It is this linear asymptotic behaviour of $\delta_{\perp}\left(k ; \kappa^{2}\right)=\mathcal{O}(k)$ for $k \rightarrow \infty$ which determines the leading $a$-term in the asymptotic $a \rightarrow 0$-expansion of (207). Here, we see one major influence of the Skyrme term on the original $O(3)-\sigma$ model, because without Skyrme-term, we have $\delta_{\perp}\left(k ; \kappa^{2}=0\right)=\mathcal{O}\left(\frac{1}{k^{2}}\right)$ for $k \rightarrow \infty$.

Besides a numerical analysis, one can also visualize the linear asymptotic behaviour of $\delta_{\perp}\left(k ; \kappa^{2}\right)$ by some simple analytic arguments. Consider the monotonically increasing series $\left(k_{i}\right)$ of real positive numbers for which $s_{\perp}\left(k_{i} ; \kappa^{2}\right) \in \mathbb{N}$. For large $k_{i}$, we may estimate $s_{\perp}\left(k_{i} ; \kappa^{2}\right) \sim\left[\kappa k_{i}\right]$ where the Gauss bracket [] denotes the integer part of a real number. With

$$
\arctan \left(\frac{1}{\kappa+1}\right) \leq \arctan \left(\frac{k_{i}}{n}\right) \leq \frac{\pi}{2}, \quad n \in\left\{1, \ldots,\left[\kappa k_{i}\right]\right\},
$$

one can use (197) to obtain

$$
\arctan \left(\frac{1}{\kappa+1}\right) \cdot\left[\kappa k_{i}\right] \leq-\delta_{\perp}\left(k_{i} ; \kappa^{2}\right) \leq \frac{\pi}{2} \cdot\left(\left[\kappa k_{i}\right]+1\right)
$$

Thus, the series $\left(-\delta_{\perp}\left(k_{i} ; \kappa^{2}\right)\right)_{i \in \mathbb{N}}$ increases linearly for $i \rightarrow \infty$. It should be possible to use the general formula for $\delta_{\perp}\left(k_{i} ; \kappa^{2}\right)$ to show that this is also true for the interpolating values of $k$.

We may therefore use the approximation

$$
-\delta_{\perp}\left(k ; \kappa^{2}\right) \lesssim-\tilde{\delta}_{\perp}\left(k ; \kappa^{2}\right)= \begin{cases}c\left(\kappa^{2}\right) & \text { for } 0<k<\alpha \\ m\left(\kappa^{2}\right) k+b\left(\kappa^{2}\right) & \text { for } k \geq \alpha\end{cases}
$$

with $m\left(\kappa^{2}\right)=\mathcal{O}(\kappa)$ for $\kappa^{2} \rightarrow \infty$. The small $\kappa^{2}$-behaviour of $m\left(\kappa^{2}\right)$ is difficult to estimate, but it is clear from the above discussion that $m\left(\kappa^{2}\right) \rightarrow 0, b\left(\kappa^{2}\right) \rightarrow 2 \pi$ for $\kappa^{2} \rightarrow 0$; the Skyrme-less limit thus changes the asymptotic $k$ behaviour of the phase shift $\delta_{\perp}\left(k ; \kappa^{2}\right)$ from a linear decrease to a constant value.

With (211), we estimate the integral (207):

$$
\begin{aligned}
h_{\perp}\left(a ; \kappa^{2}\right) \lesssim \frac{1}{\pi} \int_{0}^{\alpha} & {\left[-\tilde{\delta}_{\perp}\left(k ; \kappa^{2}\right)\right] \frac{d}{d k}\left[\ln \left(1-e^{-a \sqrt{1+k^{2}}}\right)\right] d k } \\
& +\frac{1}{\pi} \int_{\alpha}^{\infty}\left[-\tilde{\delta}_{\perp}\left(k ; \kappa^{2}\right)\right] \frac{d}{d k}\left[\ln \left(1-e^{a \sqrt{1+k^{2}}}\right)\right] d k
\end{aligned}
$$

The first integral is easy:

$$
\begin{aligned}
\frac{c\left(\kappa^{2}\right)}{\pi} \int_{0}^{\alpha} \frac{d}{d k}\left[\ln \left(1-e^{-a \sqrt{1+k^{2}}}\right)\right] d k & =\frac{c\left(\kappa^{2}\right)}{\pi} \ln \left(\frac{1-e^{-a \sqrt{1+\alpha^{2}}}}{1-e^{-a}}\right) \\
& =\ln \left(\sqrt{1+\alpha^{2}}\right)+\mathcal{O}(a) \text { for } a \rightarrow 0
\end{aligned}
$$


The second integral splits up into two parts,

$$
\frac{m\left(\kappa^{2}\right)}{\pi} \int_{\alpha}^{\infty} k \frac{d}{d k}\left[\ln \left(1-e^{-a \sqrt{1+k^{2}}}\right)\right] d k+\frac{b\left(\kappa^{2}\right)}{\pi} \int_{\alpha}^{\infty} \frac{d}{d k}\left[\ln \left(1-e^{-a \sqrt{1+k^{2}}}\right)\right] d k
$$

with

$$
\int_{\alpha}^{\infty} \frac{d}{d k}\left[\ln \left(1-e^{-a \sqrt{1+k^{2}}}\right)\right] d k=-\ln \left(1-e^{-a \sqrt{\left(1+\alpha^{2}\right)}}\right)=\mathcal{O}(\ln (a)) \quad \text { for } a \rightarrow 0 .
$$

In order to evaluate the remaining contribution, we insert

$$
0<\frac{d}{d k} \ln \left(1-e^{-a \sqrt{1+k^{2}}}\right)=\frac{k}{\sqrt{1+k^{2}}} \frac{a}{e^{a \sqrt{1+k^{2}}}-1} \lesssim \frac{a}{e^{a k}-1} \quad \text { for } k \gg 1
$$

into the first integral of (214). Using the dilogarithmic function

$$
\operatorname{dilog}(x):=\int_{1}^{x} \frac{\ln (t)}{1-t} d t
$$

we obtain

$$
\begin{aligned}
\int_{\alpha}^{\infty} k \frac{d}{d k}\left[\ln \left(1-e^{-a \sqrt{1+k^{2}}}\right)\right] d k & \lesssim \int_{\alpha}^{\infty} \frac{a k}{e^{a k}-1} d k \\
& =\frac{1}{a} \int_{\alpha a}^{\infty} \frac{\zeta d \zeta}{e^{\zeta}-1} \\
& =\frac{\pi^{2}}{6 a}+\frac{1}{a} \operatorname{dilog}\left(e^{\alpha a}\right)+\frac{1}{2} \alpha^{2} a \\
& =\frac{\pi^{2}}{6 a}-\alpha+\mathcal{O}(a) \text { for } a \rightarrow 0 .
\end{aligned}
$$

Obviously, this is the leading term in the asymptotic $a \rightarrow 0$ expansion of $h_{\perp}\left(a ; \kappa^{2}\right)$. Thus

$$
h_{\perp}\left(\beta_{T} \mu ; \kappa^{2}\right) \sim \frac{\pi m\left(\kappa^{2}\right)}{6} \frac{1}{\beta_{T} \mu} \text { for } \beta_{T} \mu \rightarrow 0 .
$$

In the Skyrme-less limit, this term vanishes $\left(m\left(\kappa^{2}=0\right)=0\right)$, and the leading $\beta_{T} \mu \rightarrow 0$ behaviour of $h_{\perp}\left(\beta_{T} \mu ; \kappa^{2} \rightarrow 0\right)$ is given by (206).

\section{References}

[1] G. t'Hooft, Phys. Rev. Lett. 37(1976)8.

[2] G. t'Hooft, Phys. Rev. D14(1976)3432.

[3] R. Rajaraman, Physics Reports 21(1975)227; R. Jackiw, Rev. Mod. Phys. $49(1977) 681$. 
[4] A. Schwartz, Phys. Lett. 67B(1977)172; M.F. Atiyah, N.J. Hitchin and I.M. Singer, Proc. Natl. Acad. Sci. U.S.A. 74(1977)2662.

[5] S.L. Adler, Phys. Rev. 177(1969)2426; J.S. Bell and R. Jackiw, Nuovo Cimento 60A(1969) 47 .

[6] A.A. Belavin, A.M. Polyakov, A.S. Schwarz and Y.S. Tyupkin, Phys. Lett. 59B(1975)85.

[7] V.A. Fateev, I.V. Frolov and A.S. Schwarz, Nucl. Phys. B154(1979)1.

[8] E. Gildener and A. Patrascioiu, Phys. Rev. D16(1977)423.

[9] C.H. Taubes, Commun. Math. Phys. 86(1982)257; 86(1082)299.

[10] N.S. Manton, Phys. Rev. D28(1983)2019; F.S. Klinkhamer and N.S. Manton, Phys. Rev. D30(1984)2212; V.A. Kuzumin, V.A. Rubakov and M.E. Shaposhnikov, Phys. Lett. 155B(1985)36.

[11] H. Kleinert, Path Integrals, World Scientific, Singapore 1990.

[12] C. Garrod, Rev. Mod. Phys. 38(1966)483.

[13] T. Matsubara, Prog. Theor. Phys. 14(1955)351.

[14] S. Habib, E. Mottola and P. Tinyakov, Phys. Rev. D54(1996)7774.

[15] A.N. Kuznetsov and P.G. Tinyakov, hep-ph/9704242.

[16] S.Yu Khlebnikov, V.A. Rubakov and P.G Tinyakov, Nucl. Phys. B367(1991)334.

[17] J.-Q. Liang an H.J.W. Müller-Kirsten, Nonvacuum pseudoparticles, quantum tunneling and metastability, in: D.H. Tchrakian (ed.), Topics in quantum field theory, World Scientific 1995.

[18] I. Affleck, Phys. Rev. Lett. 46(1981)388.

[19] A.I. Bochkarev and M.E Shaposhnikov, Mod. Phys. Lett. A2(1987)991.

[20] E. Mottola and A. Wipf, Phys. Rev. D39(1989)588.

[21] B.M.A.G. Piette, W.J. Zakrzewski, H.J.W. Müller-Kirsten and D.H. Tchrakian, Phys. Lett. 320B(1994)294.

[22] A.A. Belavin and A.M. Polyakov, JETP Letters 22(1975)245.

[23] G.M. O'Brien and D.H. Tchrakian, Mod. Phys. Lett. A4(1989)1389; Phys. Lett. B282(1992)111.

[24] G.S. Adkins, C.R. Nappi and E. Witten, Nucl. Phys. B228(1983)552. 
[25] R.P. Feynman, Statistical Mechanics, Benjamin, Reading, Mass. 1972.

[26] P. Forgács and Z. Horváth, Phys. Lett. 138B(1984)397.

[27] L.S. Langer, Ann. Phys. 41(1967)108.

[28] P. Ramond, Field Theory, Benjamin/Cummings, Reading, Mass. 1981. A.W. Wipf, Helv. Phys. Acta 58(1985)531; M.S. Volkov, hep-th/9604054.

[29] K. Funakubo, Prog. Theor. Phys. 83(1990)286.

[30] P. Forgacs, L. O’Raifeartaigh and A. Wipf, Nucl. Phys. B293(1987)559.

[31] A.G. Bochkarev and G.G. Tsitsishvili, Phys. Rev. D40(1989)1378.

[32] L. Carson, Phys. Rev. D42(1990)2853.

[33] D.H. Tchrakian, H.J.W. Müller-Kirsten and F. Zimmerschied, Phys. Lett. A197(1995)263.

[34] P.M. Morse and H. Feshbach, Methods of Mathematical Physics I, McGraw-Hill, New York 1953.

[35] S. Coleman, The Uses of Instantons, in: A. Zichichi, The Whys of Subnuclear Physics, Plenum Press, New York 1979; S. Coleman, Phys. Rev. D15(1977)2929; C. Callan and S. Coleman, Phys. Rev. D16(1977)1762.

[36] C.G. Callan, J.A. Harvey and A. Strominger, Supersymmetric String Solitons, in: String Theory and Quantum Gravity 1991: Proceedings of the Trieste Spring School, World Scientific, Singapore 1991; J.A. Harvey, hep-th/9603086.

[37] L.D. Faddeev and V.N. Popov, Phys. Lett. 25B(1967)29; L.D. Faddeev, Theor. and Math. Phys. (USSR) 1(1970)1.

[38] C. Becchi, A. Rouet and A. Stora, Phys. Lett. B528(1974)344; Ann. Phys. 98(1976)278; I.V. Tyutin, Lebedev preprint FIAN39 (1979).

[39] C.G. Callan, Jr. and D.J. Gross, Nucl. Phys. B93(1975)29; J.L. Gervais and B. Sakita, Phys. Rev. D11(1975)2943.

[40] J. Kurchan, D.R. Bes and S. Cruz Barrios, Phys. Rev. D38(1988)3309; J. Alfaro and P.H. Damgaard, Ann. Phys. 202(1990)398; H.J.W. Müller-Kirsten and J.-z. Zhang, Collective Coordinates and BRST transformations or gauge theorie without gauge fields, in: J.M. Charap (ed.), Geometry of constrained dynamical systems, Cambridge University press 1995; Jian-Ge Zhou, F. Zimmerschied, J.-Q. Liang and H.J.W. Müller-Kirsten, Phys. Lett. B365(1996)163; Jian-Ge Zhou, F. Zimmerschied, H.J.W. Müller-Kirsten, J.-Q. Liang and D.H. Tchrakian, Int. J. Mod. Phys. 11(1996)4985. 
[41] J.P. Garrahan, M. Kurczenski and D.R. Bes, Phys. Rev. D53(1996)7176; F. Zimmerschied, Fortschr. Phys. 44(1996)707.

[42] L.D. Landau and E.M. Lifshitz, Quantum Mechanics (Non-relativistic Theory), Pergamon Press, Oxford 1977. 